\title{
Application of Magnetic Resonance Imaging in Liver Biomechanics: A Systematic Review
}

\begin{abstract}
Seyed M. Seyedpour ${ }^{1,2 \dagger}$, Mehdi Nabati ${ }^{3 \dagger}$, Lena Lambers ${ }^{1,2}$, Sara Nafisi ${ }^{4}$, Hans-Michael Tautenhahn ${ }^{5}$, Ingolf Sack ${ }^{6}$, Jürgen R. Reichenbach ${ }^{7,8,9}$ and Tim Ricken ${ }^{1,2 *}$

${ }^{1}$ Institute of Mechanics, Structural Analysis and Dynamics, Faculty of Aerospace Engineering and Geodesy, University of Stuttgart, Stuttgart, Germany, ${ }^{2}$ Biomechanics Lab, Institute of Mechanics, Structural Analysis and Dynamics, Faculty of Aerospace Engineering and Geodesy, University of Stuttgart, Stuttgart, Germany, ${ }^{3}$ Department of Mechanical Engineering, Faculty of Engineering, Boğaziçi University, Istanbul, Turkey, ${ }^{4}$ Faculty of Pharmacy, Istinye University, Istanbul, Turkey, ${ }^{5}$ Department of General, Visceral and Vascular Surgery, Jena University Hospital, Jena, Germany, ${ }^{6}$ Department of Radiology, Charité - Universitätsmedizin Berlin, Corporate Member of Freie Universität Berlin, Humboldt-Universität zu Berlin, and Berlin Institute of Health, Campus Charité Mitte, Berlin, Germany, ${ }^{7}$ Medical Physics Group, Institute of Diagnostic and Interventional Radiology, Jena University Hospital-Friedrich Schiller University Jena, Jena, Germany, ${ }^{8}$ Center of Medical Optics and Photonics, Friedrich Schiller University, Jena, Germany, ${ }^{9}$ Michael Stifel Center for Data-driven and Simulation Science Jena, Friedrich Schiller University, Jena, Germany
\end{abstract}

\section{OPEN ACCESS}

Edited by:

Maurizio Schmid,

Roma Tre University, Italy

Reviewed by:

Salavat Aglyamov,

University of Houston, United States

Juvenal Ormachea

University of Rochester, United States

${ }^{*}$ Correspondence:

Tim Ricken

tim.ricken@isd.uni-stuttgart.de

tThese authors have contributed equally to this work

Specialty section:

This article was submitted to

Computational Physiology and Medicine,

a section of the journal

Frontiers in Physiology

Received: 30 June 2021

Accepted: 25 August 2021

Published: 22 September 2021

Citation:

Seyedpour SM, Nabati M, Lambers L, Nafisi S, Tautenhahn H-M, Sack I, Reichenbach JR and Ricken T (2021) Application of Magnetic Resonance Imaging in Liver Biomechanics: A Systematic Review.

Front. Physiol. 12:733393 doi: 10.3389/fphys.2021.733393
MRI-based biomechanical studies can provide a deep understanding of the mechanisms governing liver function, its mechanical performance but also liver diseases. In addition, comprehensive modeling of the liver can help improve liver disease treatment. Furthermore, such studies demonstrate the beginning of an engineering-level approach to how the liver disease affects material properties and liver function. Aimed at researchers in the field of $\mathrm{MRI}$-based liver simulation, research articles pertinent to MRI-based liver modeling were identified, reviewed, and summarized systematically. Various MRI applications for liver biomechanics are highlighted, and the limitations of different viscoelastic models used in magnetic resonance elastography are addressed. The clinical application of the simulations and the diseases studied are also discussed. Based on the developed questionnaire, the papers' quality was assessed, and of the 46 reviewed papers, 32 papers were determined to be of high-quality. Due to the lack of the suitable material models for different liver diseases studied by magnetic resonance elastography, researchers may consider the effect of liver diseases on constitutive models. In the future, research groups may incorporate various aspects of machine learning $(\mathrm{ML})$ into constitutive models and $\mathrm{MRI}$ data extraction to further refine the study methodology. Moreover, researchers should strive for further reproducibility and rigorous model validation and verification.

Keywords: liver, liver disease, biomechanics, magnetic resonance imaging (MRI), elastography, constitutive model, viscoelastic, clinical application

\section{INTRODUCTION}

The liver, an organ weighing between 1.2 and $1.5 \mathrm{~kg}$, depending on age and body mass index (BMI), in humans, is deemed the largest internal organ in adults. It accounts for $\sim 2-5 \%$ of the total of body weight (Iwai et al., 2019) and accommodates 25\% of the cardiac output (Lautt, 2009). This anatomically and physiologically complex organ (Netter, 2014) is responsible for several 
functions in the body, including synthesis and metabolism of carbohydrate, protein, and lipid, and detoxification of undesirable substances (Koeppen and Stanton, 2017). Diverse functions of the liver are attributed to a variety of cells of different embryonic origins. These cells are the bile-duct epithelial, stellate, hepatocytes, Kupffer, and sinusoidal endothelial cells and liver stem cells (Trefts et al., 2017). Two blood inflows characterize the vascular system of the liver including the portal vein and the hepatic artery, as opposed to organs such as the kidneys and brain, which are supplied by one inflow of blood. The hepatic blood outflow through hepatic veins are split into intricate treelike networks upon advancing within the liver (Lebre et al., 2017). The entire liver can be divided into functional units called liver lobules. The liver lobules have a hexagonal structure with the blood inflow at the corners. Blood flows through radially oriented capillaries, the so-called sinusoids, to the centrally located hepatic vein, which drains the blood from the liver. Each liver cell located along those capillaries, interacts with the vascular networks through the sinusoids (Lautt, 2009). The liver shape varies considerably among patients. Distinct geometric and physical properties of liver lobules cause blood circulation through liver lobules to differ among different patients. Consequently, the effects of the geometric characteristics of the liver lobules on intralobular blood flow need to be investigated, which requires an accurate representation of the liver lobules (Ahmadi-Badejani et al., 2020).

Magnetic Resonance Imaging (MRI) and computational methods both play an important role in hepatic hemodynamics studies. Four-dimensional (4D) flow MRI is an adequate tool for assessing the hepatic perfusion, and renders relevant information on spatial and temporal flow, including blood volumes and velocities (Bane et al., 2019). The latter parameters can be used to extract further hemodynamic parameters such as wall shear stress (WSS) (Roldán-Alzate et al., 2013; Wiesemann et al., 2021). Computational methods combined with WSS imaging can provide an adequate view of flow patterns under various anatomical and physiological circumstances, including portal helix and estimation of liver movement patterns (Rutkowski et al., 2019).

Pathological changes in tissues are directly linked to changes observed in the biomechanical properties of tissues such as stiffness (Evans et al., 2010; Shiina et al., 2015) and may occur in the course of various diseases such as carcinoma and malignant tumors (Evans et al., 2020), ectatic corneal disorders (Ford et al., 2011), atheroma, and calcification linked to atherosclerosis (You et al., 2015; Campo et al., 2017), cystic fibrosis-associated liver cirrhosis (Koizumi et al., 2011; Cieciura et al., 2020), and nonalcoholic fatty liver disease (NAFLD) (Mattei and Ahluwalia, 2016). Consequently, the mechanical properties of tissues hold significant diagnostic potential. Surgeons can sometimes diagnose liver tumors that had gone undetected by preoperative imaging, by laparotomy and bare touch (Elias et al., 2005). However, diagnostics via palpation is applicable only in the case of superficial organs. Furthermore, diagnosis by palpation is a subjective examination method that relies on the responsiveness and sensitivity of the practitioner's touch. Regrettably, none of the conventional medical imaging techniques, such as computed tomography (CT), MRI, and ultrasound (US), can demonstrate features that are assessed by palpation. This discrepancy has led to efforts to establish imaging techniques to evaluate the mechanical properties of the tissue quantitatively (Mariappan et al., 2010; Mueller, 2020). For example, the use of liver elastography has led to a considerable reduction in the number of liver biopsies performed to assess liver fibrosis severity (Fang and Sidhu, 2020).

Elastography examines the mechanical properties of biological tissues for diagnostic purposes. In elastography, tissue deformation is externally induced and monitored by encoding tissue motion over space and time within the organ. Various factors, including cellularity, cell types, extracellular matrix deposition, and fluid transport, alter the mechanical properties of biological tissues (Shiina et al., 2015; Sack and Schaeffter, 2018). Many elastography techniques measure stiffness without accounting for the viscoelastic, anisotropic, and nonlinear properties of most biological tissues. Consequently, the associated mechanical properties may change depending on the direction, extent, and rate of deformation. Therefore, the elastic modulus of biological tissues is a complex quantity consisting of a storage modulus and a loss modulus that represent elasticity and viscosity, respectively. The viscosity component shows the damping behavior, in which the strain rate changes with time resulting from the loss of strain energy. Mathematical models such as those proposed by Maxwell, Voigt, Zener, Jeffreys, as well as a fractional Zener model have been devised to predict the stress-strain dynamics of viscoelastic materials (Klatt et al., 2007; Low et al., 2016; Holm, 2019). The elasticity modulus is described per the type of encountered stress as Young's modulus (E), Shear modulus $(\mu$ ), or Bulk Modulus (K) (Low et al., 2016). There are different techniques for elastography, all mainly based on US, MRI, and optical coherence tomography methods. In addition to imaging systems, these techniques differ in the source of externally induced stress, which can be static, quasi-static, or dynamic, the latter with the distinction of transient or time-harmonic (Gao et al., 1996; Manduca et al., 2001; Sarvazyan et al., 2011; Wells and Liang, 2011; Vappou, 2012; Low et al., 2016; Ormachea and Parker, 2020; Li et al., 2021). In general, elastography methods apply mechanical stress or stimulation to the tissue, monitor the tissue response to the induced stimulation, and employ this image-encoded response to reconstruct parameters that denote mechanical properties. Meanwhile, elastography techniques are in routine clinical use for the detection of liver fibrosis (Angulo et al., 2007; Yoneda et al., 2007, 2010; Castera et al., 2008; Harrison et al., 2008; Sumida et al., 2011, 2019; Imajo et al., 2016). Other emerging clinical applications of elastography include the diagnosis of cancer (prostate, breast, liver, pancreas) (Thomas et al., 2006; Garteiser et al., 2012; Fischer et al., 2013; Asbach et al., 2020; Zhu et al., 2020), kidney diseases (Garcia et al., 2019; Lang et al., 2019) thyroiditis, arterial plaque evaluation, arterial stiffness (Kolipaka et al., 2012; Schaafs et al., 2019), deep-vein thrombosis evaluation (Hoang et al., 2017; Mumoli et al., 2018), and measurements of human corneal biomechanical properties using optical coherence elastography (OCE) (Wang and Larin, 2015; Lan et al., 2020). Magnetic resonance elastography (MRE) (Muthupillai and Ehman, 1996) is potentially more accurate 
and less operator dependent than ultrasound elastography (Bonekamp et al., 2009; Faria et al., 2009; Serai et al., 2015; Barr, 2018; Lupescu et al., 2020). One reason for this is the use of timeharmonic mechanical vibrations in a frequency range between 20 and $300 \mathrm{~Hz}$, which can illuminate the full body by shear waves. Furthermore, the excellent soft-tissue image contrast of MRI, which is fully three-dimensional and free of acoustic shading, adds to the high precision and potential accuracy of MRE (Hirsch et al., 2017). MRE uses modified phase-contrast MRI sequences to encode three-dimensional wave fields which are then converted into maps of the complex shear modulus (elastograms) by an inverse algorithm (Venkatesh et al., 2013, 2015; Serai et al., 2015; Tang et al., 2015; Srinivasa Babu et al., 2016; Tzschätzsch et al., 2016; Lupescu et al., 2020; Manduca et al., 2020). The advantages of MRE include the facility to examine the entire liver and convenient application in screening of obese or injured patients (Venkatesh et al., 2013, 2015; Serai et al., 2015; Tang et al., 2015; Srinivasa Babu et al., 2016; Lupescu et al., 2020).

MRI may also serve to define the shape and geometry of the liver in addition to the hemodynamics and the properties of the liver tissue and components. MRI can be applied to reconstruct the liver surface and blood vessels, including portal vein and the branches of the hepatic veins. Moreover, the vein diameters and lengths can be attained from the segmentation data.

The ever-increasing number of MRI-assisted biomechanical studies on the liver underscores the need to integrate existing evidence into research. Systematic reviews address this need by assembling prior research based on standard strategies. Such studies, which are common in medicine (Uman, 2011), may serve as adequate tools for researchers who perform studies in the field of liver biomechanics for gaining a better understanding of the later stages ahead. The main objective of this study is to present a comprehensive overview of current studies on liver biomechanics with the aid of MRI. The secondary objective is to examine the structural models adopted in liver MRE. Furthermore, we intend to propose relevant suggestions for the better use of liver biomechanics in clinical trials.

\section{MATERIALS AND METHODS}

To design a protocol for the present review, we employed the Cochrane guideline (Higgins et al., 2019) and PRISMAP checklists (Shamseer et al., 2015) so that the standards of the protocol were satisfied. Although the present review is not of Diagnostic Test Accuracy (DTA) or interventional type (Higgins et al., 2019), the standards complied. Moreover, a modified protocol for implementing review works and evaluating approaches was developed. Given the current review's characteristics, the protocols were modified by making a change in the elements and turning them into research object, object modeling, properties, and tools. The reason is that there are no participant, intervention, comparator, and outcome (PICO) elements available other than in interventional reviews (Higgins et al., 2019). In the latter type of reviews, the research object is described as the tissue or organ under investigation. Imaging techniques, like MRI, CT, and X-Ray, are determined by tools. By object properties, material properties, constitutive relationships, and geometry are meant. In the modeling process, numerical simulation approaches such as the Finite Element Method (FEM), elastic and viscoelastic models for elastography, and the Finite Volume Method (FVM) are implemented. A twostep key term search was employed for finding peer-reviewed research articles related to the topic of the present review. The pre-specified selection criteria were used for designing the basic strategy. As the first criterion, we selected the papers that had employed MRI as their research tool. The second criterion was to select those articles that had investigated the liver from a biomechanical perspective including liver material properties, hemodynamics, and shape and geometry. Although the viscoelastic properties of focal lesions and model identification in liver tumors are extremely interesting, this review focuses on the properties of liver tissue under healthy and chronic disease conditions. We did not exclude liver lesions per se from our review, but could not elaborate on this point due to limited data regarding mechanical modeling in liver tumors in vivo.

\subsection{Inclusion Criteria}

Based on the elements defined above, the criteria were classified into four classes as follows: The first class includes imaging techniques that are based on MRI, including magnetic resonance imaging "MRI," magnetic resonance elastography "MRE," "2D-MRI," "4D-MRI," "3D-MRI," nuclear magnetic resonance imaging "NMRI," and "DT-MRI." The second class includes studies that used the terms "Hepatic," "Liver," "Liver lobule," and "Blood perfusion." The third class contains properties of objects, including "Material property," "Biophysics," "Biomechanics," "Boundary condition," "Geometry," and "Mechanical behavior." The fourth class pertains to numerical plans such as finite difference method "FDM," "Mesh-free," finite element method "FEM," finite volume method "FVM," computational fluid dynamic "CFD," "Modeling," "Simulation," and artificial neural network "ANN," which were employed to study the mechanical responses of the liver. As a result, no study was missed due to the constraints on the model types. In this way, we designed a search strategy that retrieved all articles that dealt with liver modeling. Ultimately, the search process was limited to studies that investigated the liver from an engineering point of view. Review papers were not included, only original research papers. To assess all relevant research papers, only papers published during the period 1980-2020 were searched and included. In terms of the used MRI type, no paper was excluded. Only papers in German or English languages were included.

\subsection{Search Strategy}

Using the predetermined selection criteria, we designed an inclusive search strategy for the retrieval of all related articles, and various sources were searched, ensuring that no article was overlooked (cf. Appendix B). Additionally, a list of equivalent terms was prepared and included in the search strategy. For initiating the search, a primary search was performed in the electronic databases on October 25, 2020. Web of Science, 

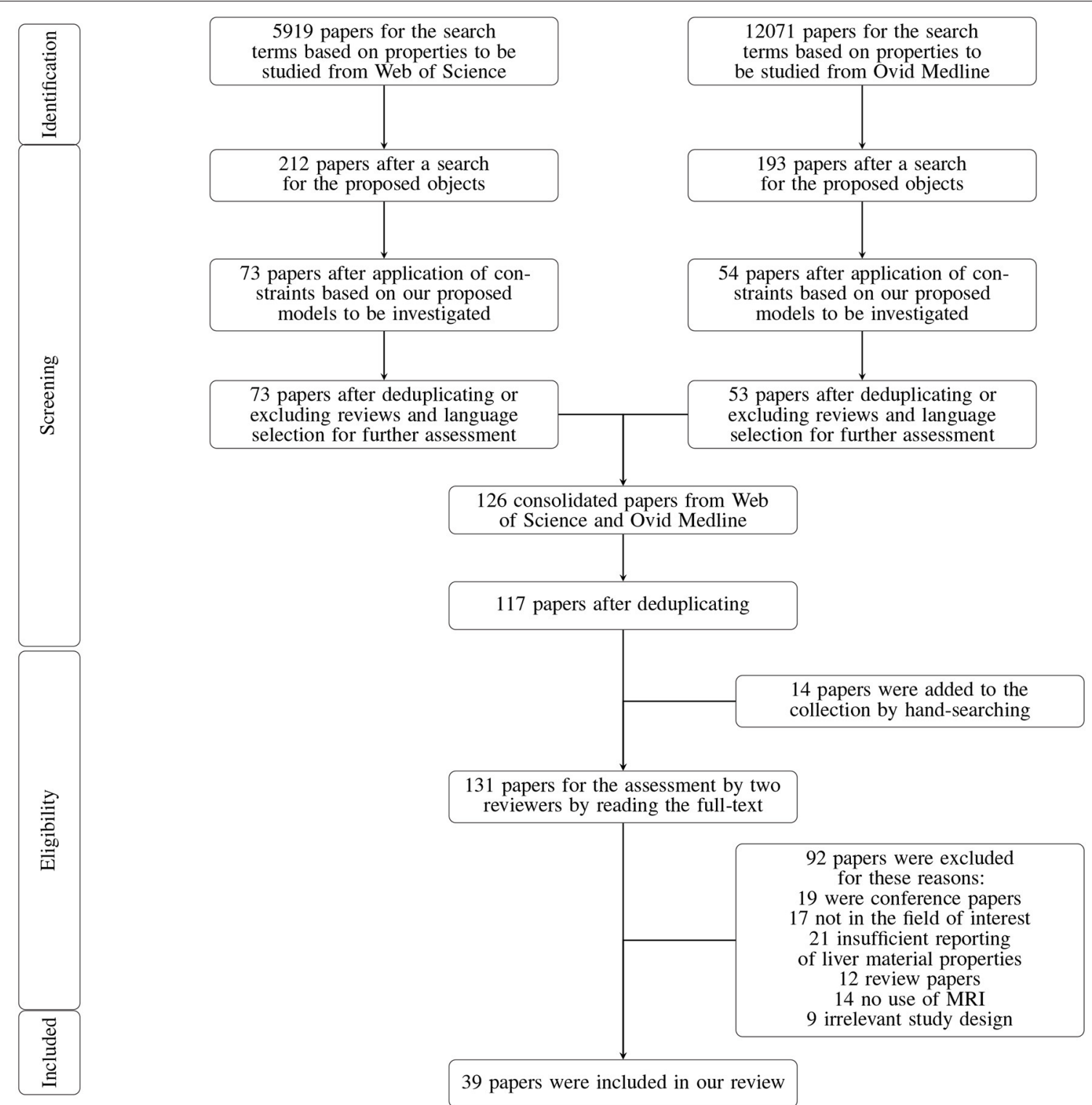

FIGURE 1 | Flowchart of the screening process.

Springer, and Medline (Ovid SP) (January 1980-October 2020) were used as search engines. The last search was conducted on December 10, 2020. Moreover, using key terms given in Appendix B, other electronic datasets, such as Wiley, Taylor \& Francis, ScienceDirect, and Pubmed were searched. In the next step, a manual search was performed in the references so that any related studies that may not have been found using the search strategy were included.

\subsection{Selection of Papers}

The identified papers were selected based on pre-specified phases. First, papers that were not relevant in terms of titles and abstracts were excluded. Two reviewers assessed the full-text of the remaining papers for obtaining the final list of papers that were included in the review. The flow diagram of the strategy designed for the paper selection is demonstrated in Figure 1.

\subsection{Extraction of Data}

Data were extracted from the included studies by two reviewers, who collected the final data in a previously designed proforma (cf. Appendix C). The data collected in the proforma contained the following properties: The patient or object under study, MRI type, application of MRI, modeling field, the purpose of research, constitutive model, and research clinical application.

\subsection{Quality Assessment}

To assess the quality of the included papers, some questions were designed according to previous methods applied for 
quality assessment in standard protocols, like PRISMA-P and Cochrane (Shamseer et al., 2015; Higgins et al., 2019). The issues proposed in the standard guideline were modified following this context. Next, questionnaires used in systematic review articles of biomechanics studies (Riva et al., 2013; Hindle et al., 2019) were utilized for preparing a questionnaire (cf. Appendix D). This set of questions includes five main sections, including research objectives, research design, result presentation, bias assessment, and ethical issues. Each question receives a score of $0,1,2$, or not applicable. Two reviewers separately conducted the assessments. Three groups of items, i.e., the items related to research objectives, research design, and assessment of bias, were regarded as critical as agreed by both reviewers. Thus, the reviewers assessed the quality of the studies considering these items. By definition, a high-quality study was defined as a study that had a maximum of one zero score in the critical items. Studies that received two zero scores on these items and a maximum of one zero score on the non-critical items were considered average-quality papers. Papers with more than or equal to two zero scores in critical items, and more than one zero score in non-critical items were considered papers with low quality.

\section{RESULTS}

\subsection{Results of the Systematic Search}

The search through the electronic databases Ovid Medline and Web of Science resulted in 17,990 papers. The liver was the research object in 405 of the papers. Then, constraints were applied based on the models under study, leading to 127 studies. Review papers, duplicate papers, and papers in languages other than German or English were excluded after applying the limitations in the search platforms, resulting in 117 papers remaining. After inclusion of 14 studies from the hand-search, 131 papers were assessed by two reviewers by reading the fulltexts. Eventually, 46 papers were selected for quality assessment and data extraction. The detailed flowchart of paper selection can be seen in Figure 1. Short descriptions of the reviewed papers are presented in Appendix E.

\subsection{MRI Types and Application}

MRI scanners can be classified according to the strength of the magnetic field (McRobbie et al., 2017). Low-field MRIs (0.3 $\mathrm{T}$ ) are much less expensive, provide better patient access. The main disadvantage of $0.3 \mathrm{~T}$ is the reduced signal-to-noise ratio compared with High-field MRI systems (Konar and Lang, 2011). The most common field strength used in clinical applications are 1.5 and $3 \mathrm{~T}$. Assessment of the liver using 1.5 or $3 \mathrm{~T}$ MRI has both advantages and disadvantages. The Signal to Noise Ratio (SNR) is higher with $3 \mathrm{~T}$ systems, resulting in improved image quality and shorter scan time. Further advantages of $3 \mathrm{~T}$ compared to $1.5 \mathrm{~T}$ are higher in-plane spatial resolution and thinner slice thicknesses. Almost all of the reviewed papers used 1.5 or $3 \mathrm{~T}$ MRI systems, but it is expected that as the availability of $7 \mathrm{~T}$ MRIs systems becomes more widespread. Since the SNR of a $7 \mathrm{~T}$ system is slightly higher than a $3 \mathrm{~T}$ system, and extra SNR leads to higher resolution images (McRobbie et al., 2017). Furthermore,
$7 \mathrm{~T}$ relaxation values have a slightly more significant difference from $3 \mathrm{~T}$ values when assessing differences between healthy and unhealthy liver in vivo. Of the 46 studies, 21 used a $1.5 \mathrm{~T}$ scanner (Kruse et al., 2000; Brock et al., 2005; Klatt et al., 2007; Salameh et al., 2007; Asbach et al., 2008, 2010; Chen et al., 2011; Wang et al., 2011; Garteiser et al., 2012; Godfrey et al., 2012; Kamphues et al., 2012; Leclerc et al., 2013, 2015; Courtecuisse et al., 2014; Lee et al., 2014; Tzschätzsch et al., 2014; Reiter et al., 2018, 2020; Hudert et al., 2019; Shahryari et al., 2019; Dzyubak et al., 2021), six a 3 T scanner (Roldán-Alzate et al., 2013; Monti et al., 2014; Ning et al., 2018; Rutkowski et al., 2018, 2019; Amili et al., 2019), and four studies a 7 T scanner (Salameh et al., 2009; Riek et al., 2011; Reiter et al., 2014; Ronot et al., 2014). Two studies used both 1.5 and $3 \mathrm{~T}$ (Lee et al., 2010; Motosugi et al., 2019), and one study used 0.3 and $3 \mathrm{~T}$ (Tomita et al., 2018). Twelve studies (Hariharan et al., 2007; Clarke et al., 2011; Lara et al., 2011; Zhang et al., 2013, 2014; Lu and Untaroiu, 2014; Tang and Wan, 2014; Idkaidek and Jasiuk, 2015; Stoter et al., 2017; Ma et al., 2019; Eaton et al., 2020; Gidener et al., 2020) did not mention the magnetic field strength of the used MRI scanner. Of the 46 studies, six used MRI to determine the geometry and surface of the liver, eight used MRI for hemodynamic studies of the liver, four used MRI for motion and deformation capture of the liver, and 29 used MRI in order to study the elastography and tomoelastography of the liver. Further details of the MRI acquisitions and applications are summarized in Appendix F.

\subsection{Viscoelastic Models Used in the Liver MRE}

A variety of viscoelastic models were used in the liver MRE studies in the reviewed papers. Of the 46 reviewed papers, 29 papers performed MRE on the liver. Four papers studied the liver with linear isotropic elastic models (Kruse et al., 2000; Tang and Wan, 2014; Tzschätzsch et al., 2014; Leclerc et al., 2015), which provide a simple and straightforward relation between stress and strain. Among them, one paper compared the elastic results with the Voigt model (Tzschätzsch et al., 2014) as the simplest viscoelastic model. Four papers used the Voigt model (Klatt et al., 2007; Salameh et al., 2007; Leclerc et al., 2013; Tzschätzsch et al., 2014) and three of them compared the results with the other models. Among these papers, Klatt et al. (2007) compared Voigt, Maxwell, Zener, Jeffereys, and fractional Zener. In three studies, the liver was described with the Zener model (Klatt et al., 2007; Asbach et al., 2008; Tomita et al., 2018), and in four papers the liver was modeled with the spring pot model (Riek et al., 2011; Kamphues et al., 2012; Leclerc et al., 2013; Reiter et al., 2014). One paper studied the liver with an exponential model (Clarke et al., 2011) for large strain, while three papers did not mention their model (Salameh et al., 2009; Garteiser et al., 2012; Ronot et al., 2014). The material models reported in the reviewed articles have been categorized and are listed in Appendix G. MRE at higher field strengths, such as at $3 \mathrm{~T}$, has become a routine application in clinical imaging for the detection of liver fibrosis. In particular, spin-echo MRE sequences are used to mitigate the strong signal $\mathrm{T}^{*}$ decay in the liver at higher field strengths. However, it should be noted that $1.5 \mathrm{~T}$ scanners, although increasingly less popular 
for clinical MRI, are advantageous for liver imaging. Specifically, MRE of livers in the presence of iron overload benefits from less signal decay at $1.5 \mathrm{~T}$ than at $3 \mathrm{~T}$. The relatively narrow range of frequencies $(10-100 \mathrm{~Hz})$ in MRE is certainly an issue. Therefore, studies relied on simple two-parameter viscoelastic models do not overfit shear modulus dispersion curves. Although two-parameter fits based on a typical set of four frequencies acquired by MRE can significantly vary with noise and data quality, the main tendency of viscoelastic parameter changes has been consistent across studies and etiologies. Furthermore, eight independent measures are fitted by only two parameters if complex modulus data are analyzed, providing sufficient stability for viscoelastic modeling.

\subsection{Clinical Application}

Combining liver biomechanics with MRI techniques could be beneficial in many medical applications.

4D MRI can provide valuable knowledge to understand hepatic hemodynamics, which is important for treating congenital heart defects (Lara et al., 2011). Moreover, the incorporation of circulation in all hepatic vessels indicates a higher potential for clinical use in the hepatic perfusion model (Ma et al., 2019). Computational simulations using a combination of $4 \mathrm{D}$ MRI flow and particle image velocimetry can reveal the relationships between portal vessel geometry, flow structure development, and blood flow distribution in distal hepatic vasculature (Rutkowski et al., 2019). In pre-treatment settings, MRI images can be registered using FEM-based deformable image registration, which allows the visualization and contouring of the liver on each image to direct the deformation of other regions of interest. This may be particularly useful for MR registration, where the tumor may have different image characteristics for each modality, and the deformation of the image can compromise geometric accuracy (Brock et al., 2005). Viscoelasticity-based MRE can be a diagnostic tool for detecting the disease process, such as cirrhosis (Asbach et al., 2008), fibrosis (Salameh et al., 2007), or fibrosis in chronic hepatitis $\mathrm{C}$ transplant patients (Kamphues et al., 2012), and might become a useful alternative method to liver biopsy. Furthermore, liver elastography might have a role in the early detection and treatment assessment of nonalcoholic steatohepatitis in patients (Salameh et al., 2007). In addition, MRE protocols could be applied for the follow-up of the effects of treatments (Leclerc et al., 2015).

\subsection{Objects of Reviewed Papers}

Of the 46 papers, 30 studied human liver, while 12 dealt with animal liver (Kruse et al., 2000; Hariharan et al., 2007; Salameh et al., 2007, 2009; Clarke et al., 2011; Riek et al., 2011; Courtecuisse et al., 2014; Reiter et al., 2014; Ronot et al., 2014; Tang and Wan, 2014; Idkaidek and Jasiuk, 2015; Ning et al., 2018). Phantoms and experimental models as liver-mimicking material were studied in three papers (Lara et al., 2014; Leclerc et al., 2015; Amili et al., 2019), while two papers investigated both the human liver and phantoms (Lee et al., 2010; Tomita et al., 2018), and one paper studied human and animal liver (Reiter et al., 2014). Thirty-two articles (69.56\%) examined liver in vivo, while four (8.69\%) studied liver ex vivo. Eight (17.39\%) studies were performed in vitro, and two papers reported both in vitro and in vivo experiments. Eight studies investigated both genders and 11 studies did not mention the gender of the patients. Six studies included only healthy subjects. However, there were 10 studies in which healthy and unhealthy subjects were examined at the same time. Twenty studies investigated liver diseases. Detailed study type, population, and diseases are summarized in Appendix $\mathrm{H}$.

\subsection{Quality Assessment}

In accordance with the protocol mentioned in the Materials and method section, the quality assessment was conducted separately by two reviewers (SMS, MN). The results are summarized in Appendix I. There were 32 high-quality papers, three medium-quality papers, and eleven low-quality papers among the retrieved papers. Of the low-quality papers, seven did not mention any hypotheses and had fewer than three participants. Two of the medium-quality papers had the same problems; however, due to fewer problems with the non-critical criteria, they were ranked medium-quality papers rather than low-quality papers.

\section{DISCUSSION}

\subsection{Hepatic Hemodynamic Measurement Using 4D Flow MRI}

Hemodynamic parameters in splanchnic vessels, such as volume, pressure, velocity, and stress, can presumably depict vascular changes caused by liver diseases such as cirrhosis and portal hypertension, hepatocellular carcinoma, steatosis, and vascular occlusion (e.g., Budd-chiari-Syndrom; Scheinfeld et al., 2009; Zhang et al., 2011; Stankovic et al., 2013). Most hepatic diseases cause a heterogeneous lobar distribution hypothesized due to imbalanced distribution of blood flow between portal venous branches (Lara et al., 2011; Roldán-Alzate et al., 2015). 4D flow MRI has been developed as a beneficial non-invasive tool for the assessment of hepatic hemodynamics (Bane et al., 2019). Furthermore, before and after liver surgery, results of $4 \mathrm{D}$ flow MRI provide an insight into hemodynamic changes resulting from resection. The use of $4 \mathrm{D}$ flow MRI can serve as a source for simulation conditions and a benchmark for numerical blood flow simulations (Rutkowski et al., 2018). Since the results of pre-surgical 4D flow MRI alone cannot reliably provide information about the liver hemodynamics after surgery, numerical simulations are required to predict the chance of surgical success.

Although 4D flow MRI can measure flow parameters in any vessel contained in the acquired volume by measuring not only a volumetric velocity map but also considering crosssectional area changes in the vessel of interest during the cardiac cycle, it is challenging because of the need for large volumetric coverage, high spatial resolution, sensitivity to a wide range of flow velocities, the need for respiratory gating and the need for short scan times (Roldán-Alzate et al., 2015). Therefore, the use of cartesian-based 4D flow approaches has been recommended for portal vein research (Stankovic et al., 2014). 
Liver stiffness measured by elastography in MRI and ultrasound has been shown to be sensitive to blood flow and perfusion, which can be altered by physiological variations such as water intake and respiratory maneuvers. Because hemodynamic variations are a confounding factor for measured shear stiffness, it is recommended that elastography studies be performed under standardized fasting and breathing conditions to improve the reproducibility and diagnostic accuracy of the method. A model of elastic behavior is developed based on the flow of viscous fluids through the extensive network of tissue microchannels in response to applied stress to study the functional (non-pathological) of the liver (Parker, 2014). Furthermore, the integrated model parameters are directly linked to tissue vascularity and fluid channels in order to predict an abnormal condition (Parker et al., 2016).

\subsection{Perfusion MRI}

Perfusion MRI evaluates the microcirculatory state of the liver parenchyma and lesions, which can be used to identify liver metastases, evaluate antiangiogenic therapy efficacy, determine tumor viability after anticancer therapy or ablation, and diagnose liver cirrhosis and its seriousness (Thng et al., 2010; Hariharan et al., 2013; Stoter et al., 2017). Perfusion MR parameters can be derived using model-free or model-based techniques which analyze the contrast concentration-time curve in focal liver lesions or liver parenchyma, derived from the dynamic contrastenhanced MRI images (Moawad et al., 2020). The model-free method is based on capturing the rate of tissue enhancement in relation to contrast passage through the tissues. To calculate the hepatic perfusion index, perfusion parameters are derived from the maximal slope of the time-to-intensity curves of the hepatic artery and portal vein (Moawad et al., 2020). The model-based approach includes curve fitting of a dual-input single-compartmental model. The parameters obtained from the model measure the agent's pharmacokinetic distribution in physiological terms such as arterial and portal venous blood flow, distribution volume, and mean transit time. However, it should be mentioned that the model-based methods are based on certain assumptions, which may influence the precision of the derived parameters (Zhou et al., 2020). Moreover, the perfusion profile of the liver, which can be computed with the diffuse interface method, reproduces key properties of the flow, such as regions of higher and lower flow velocities due to local narrowing or widening of vessel cross-sections (Stoter et al., 2017).

\subsection{Liver Segmentation in MRI}

Reliable liver segmentation has particular relevance in the planning (Chlebus et al., 2018), monitoring, and treatment of various liver diseases such as diffuse liver disorders, liver cancer (López-Mir et al., 2014), and liver transplantation (Goldaracena and Barbas, 2019). In comparison to other organs, liver segmentation is more complicated. These complications arise because of the liver's extremely complex structure and adjacency to other tissues. Furthermore, the liver is affected by a variety of pathologies that alter its mass, signal strength, and architecture (Gotra et al., 2017). Liver segmentation strategies include manual, semi-automated, and fully automated. In manual segmentation, the operator performs liver segmentation on sequential MRI slices by contouring pixels around the boundaries or inpainting the liver parenchyma. This approach is strongly dependent on operator interaction. Semi-automated segmentation techniques, such as intensity-based techniques and graph cut, have to be initialized by the operator. The rest of the optimization is performed by the algorithm. These methods often rely on a variety of interactions. For typical datasets, fully automatic segmentation strategies need minimal operator feedback. However, in special cases, they can require manual modification. Since liver segmentation is labor-intensive and time-consuming, deep learning (DL) has been applied for automated liver segmentation (Chen et al., 2020).

\subsection{Viscoelastic Models in the Liver MRE Studies}

MRE depends on the stress-strain response of soft tissues exposed to external stimuli. This response can be modeled by combining elastic and viscous elements that characterize the particular rheological behavior of the tissue. Viscoelastic parameters of tissues can be measured using MRE with different frequencies, which enables the deduction of the dispersion of the wave propagation speed and the coefficient of wavedamping (Kruse et al., 2000; Klatt et al., 2007; Sack et al., 2008). MRE data can be used to determine different constitutive parameters of different rheological models, including the models of Voigt, Maxwell, Zener, Jeffreys, and the fractional Zener model (cf. Figure 2). These models consist of three independent constitutive parameters, namely spring, dashpot, and spring pot. The Voigt and Maxwell models both contain a spring and a dashpot. The Zener and Jeffreys models are extensions of the Voigt model. While the Zener model has an additional spring connected in series with the dashpot compared to the Voigt model, the model of Jeffreys has an additional dashpot in series with the Voigt model. The fractional Zener is also an extension of the Zener model, in which a spring pot element replaces the dashpot. The spring pot represents an interpolation between purely elastic and purely viscous behavior, which can be reduced to a spring and a dashpot. The Zener model can better reproduce the dispersion of experimental data, while the fractional Zener model can increase the variation of the results (Sack et al., 2008). The Voigt and Maxwell models are the simplest viscoelastic models. The Voigt model is often used for solids, while the Maxwell model is used for fluids (Catheline et al., 2004). Of the simplest viscoelastic models, the Voigt model tends to be best suited to the viscoelastic parameters of soft tissues (Catheline et al., 2004; Salameh et al., 2007). The Jeffreys model is more suitable in the study of the fluids (Sack et al., 2008; Leclerc et al., 2013). The Zener model can better reproduce the dispersion of experimental data, while the fractional Zener model can increase the variation of the results (Riek et al., 2011). Although the structure geometry parameter $\alpha$, which is dimensionless power law exponent (cf. Appendix G), does not change with the stage of fibrosis in the liver, $\mu$ is the only parameter to be evaluated in order to determine the stage of fibrosis in clinical practice. Hence, the robust spring pot model is especially suitable for the 


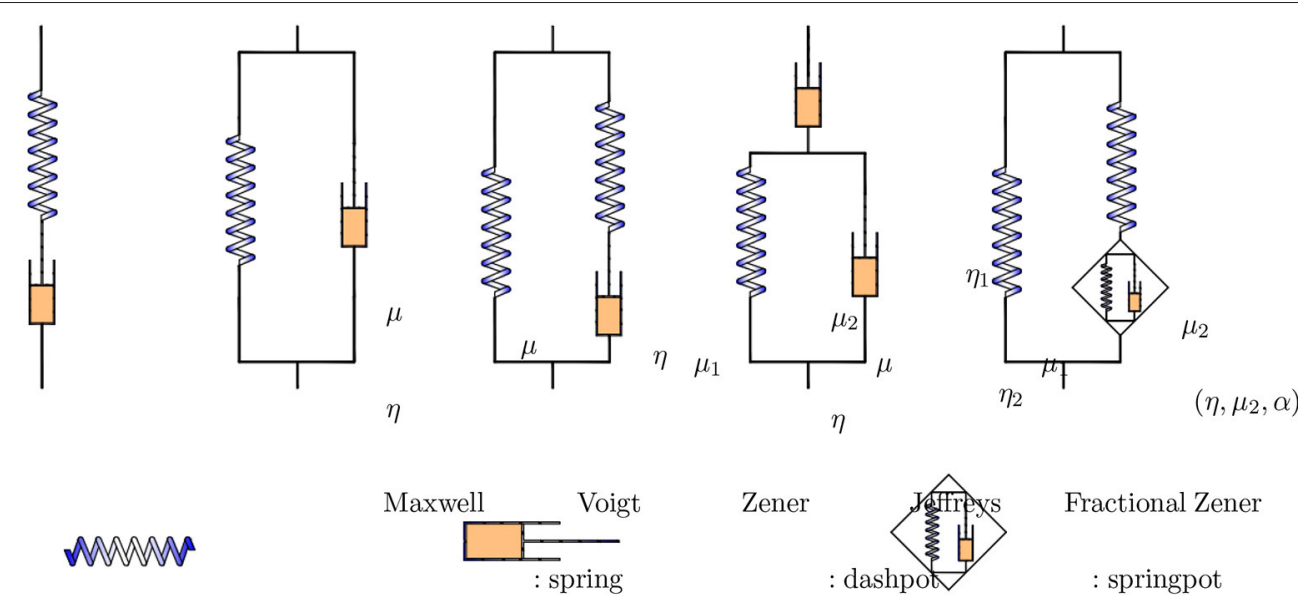

FIGURE 2 | Common viscoelastic models used in liver MRE study.

study of liver fibrosis in comparison with the Voigt and Maxwell models (Asbach et al., 2010). More details on the attenuation of shear waves in viscoelastic powerlaw media can be found in Holm (2019).

The constitutive parameters $(\eta, \mu, \alpha)$ can be related to complex modulus $G^{*}$ using various viscoelastic models. $G^{*}$ consists of a real part $\left(G^{\prime}\right)$, which denotes elasticity, and an imaginary part $\left(G^{\prime \prime}\right)$, which denotes viscosity. $G^{\prime}$ is determined by the mechanical energy recovery to the material's elastic properties, while $G^{\prime \prime}$ is connected to the inherent mechanical friction of the viscous properties given to the tissue (Meyers and Chawla, 2008). The magnitude of the complex shear modulus $\left(\left|G^{*}\right|\right)$ is often referred to as stiffness, but since it represents the geometric mean of $\left(G^{\prime}\right)$ and $\left(G^{\prime \prime}\right)$, it combines both elastic and viscous properties. In this respect, the connotation of $\left(\left|\mathrm{G}^{*}\right|\right)$ as stiffness is a colloquial term that conveys the subjective haptic impression of whether a solid tissue with predominantly elastic properties behaves stiffer than surrounding tissues. The phase angle $(\varphi)$ of $\mathrm{G}^{*}$ illustrates fluidity. $\varphi=0$ represents pure solid, while $\varphi=\frac{\pi}{2}$ is representative of pure fluids. $\varphi<\frac{\pi}{4}$ indicates solid dominant tissues, while $\varphi>\frac{\pi}{4}$ illustrates fluid dominant tissues (Hirsch et al., 2017; Shahryari et al., 2019).

Alterations in the hepatic stiffness are associated with changes in solid-tissue properties, and fluid-tissue properties are associated with changes in the stiffness of liver tumors. Hence, fluidity could be a biomechanical marker in order to detect and distinguish liver tumors (Shahryari et al., 2019). Tomoelastography is an in vivo method for quantitative mapping of the solid-fluid properties of soft tissues (Hudert et al., 2019; Shahryari et al., 2019). Furthermore, tomoelastography can be used for the evaluation of pediatric NAFLD (Hudert et al., 2019).

Studies using ultrasound-based and MR elastography have shown that the liver stiffens with food and water intake or portal hypertension. Parker (2014) proposed a microchannel flow model which can explain the stiffening of liver tissue due to changes in temperature or salinity of the vascular fluid. Moreover, Parker et al. have theoretically investigated the influence steatosis and steatohepatitis on the liver's viscoelastic properties by simulating composite materials of oil-gelatin mixtures (Parker et al., 2018). The results nicely demonstrate the sensitivity of frequency dispersion of viscoelastic parameters to the fat content in the liver. However, since our review work systematically analyzes viscoelastic modeling of in vivo liver tissue, we cannot include phantom investigations without moving the topic of this review article to an entirely new area of focus.

\subsection{Magnetic Resonance Poroelastography (MRPE)}

The shear module achieved by reconstructing the biological soft tissue, based on common MRE, cannot be descriptive of the distribution of true solid matrix parameters (Perriñez et al., 2008). Furthermore, tumors tend to have shear moduli between one and two orders of magnitude higher than surrounding tissue, which can be interpreted as poroelastic material rather than viscoelastic material (Sowinski et al., 2020). Magnetic resonance poroelastography (MRPE) allows the analysis of tissue-poroelastic behavior by distinguishing the mechanical response of the solid matrix from the free extracellular fluid (Perriñez et al., 2008). MRPE determines the time-harmonic pore-pressure field, which is caused by external vibration, while MRE is based on the single-phase elastographic image reconstruction (Perriñez et al., 2010). MRPE can adequately describe the mechanical properties of the liver and its poroelastic deformation. Hence, MRPE could provide more representative mechanical properties of the liver for the simulation of liver disease and liver transplantation. PMRE provides fluidrelated quantities, including pore-fluid pressure and hydraulic conductivity (Tan et al., 2017), but it cannot provide pressure boundary conditions (PBCs).

\subsection{Clinical Application}

One of the main objectives of the reviewed studies is how physicians can use the results of MRI-based biomechanical researches as medical diagnostic methods in clinical trials. 
Although most of the papers included in our review provide a view on the clinical application, not all of them report on the actual applications. Besides, among the studies included in our review, only 12 studies recruited healthy and sick subjects. These studies provide useful information on the value of providing control groups in order to conclude that the procedure in the clinic can be used. Since the clinical use of an intervention depends largely on the quantity of the evidence, the reproducibility of results on a higher number of volunteers would help expand the use of simulations in clinical settings.

\subsection{Continuum-Biomechanical Models of the Liver}

Soft tissues, especially the liver, are materials whose mechanical response is characterized by complex layer heterogeneity, anisotropy, and non-linearity. Although several constitutive models of the liver have been developed, a realistic, comprehensive constitutive model covering the entire spectrum of the liver's complex response is still not available. Improved constitutive models could enable the simulation of the liver deformation and become increasingly valuable in many areas of biomedical engineering. Such constitutive models for the liver, combining advanced imaging and computational biomechanics, can help in medical and industrial development. The liver has been treated as purely macroscopic with a single scale model as linear viscoelasticity material in the earliest models (Liu and Bilston, 2000). Although such a model cannot account for the non-linearity of the mechanical response, the liver can be considered a single-phase viscoelastic material. The effect of fluid pressure on tissue stiffness has been simplified in the effective modulus to describe its time-dependent response. Moreover, the liver has been modeled as nonlinearly hyperelastic (Schwartz et al., 2005). In addition to macroscopic models, multiscale models considered the coupling of the microstructure to the macrovascular structure (Mescam et al., 2009). However, the drawback of single-phase models is that they do not reflect the effect on the microscale of poroviscoelasticity caused by the frictional combination of tissue and blood components. Poroviscoelastic (PVE) models generalize the linear biphasic models that include flow-independent viscoelasticity in the solid-phase description. Different kinds of model approaches exist for fluid-saturated porous solids as the Biot theory (BT) (Malandrino and Moeendarbary, 2019), mixture theory (MT) (Drumheller, 1978; Klisch et al., 2003; Patki and Costanzo, 2019), or Theory of Porous Media (TPM) (de Boer, 2012; Ricken et al., 2015). The BT disadvantage compared to the MT or TPM is its thermodynamic inconsistency and is, therefore, inadequate for further development such as growth.

\subsection{Liver Diseases Considered in Mathematical Models}

Computer-aided calculations have already been developed for a variety of liver diseases. Liver fibrosis is characterized by highly increased accumulation of collagen and thus scarred tissue of the liver. Several models have been presented to describe the development of fibrotic tissue due to various causes, e.g., treatment with carbon tetrachloride $\left(\mathrm{CCl}_{4}\right)$ (Dutta-Moscato et al., 2014), chronic Hepatitis B (Wei et al., 2019), and Hepatitis C (Lara et al., 2014) or fibrosis in general (Friedman and Hao, 2017). Cirrhosis is an aggravated and non-reversible form of fibrosis. To investigate perfusion changes in cirrhotic livers, CFD can be used (Peeters et al., 2015). Another method to calculate changes in perfusion in normal, fibrotic, and cirrhotic livers is the porous media approach ( $\mathrm{Hu}$ et al., 2017). Inherited hepatic diseases can also be studied using computational models. Hemochromatosis, which is caused by an overload of iron in the human body, has been simulated by focusing on the iron metabolism in the liver (Mitchell and Mendes, 2013). Suffering from NAFLD, fat vacuoles are stored in the hepatocytes. This disease, which occurs due to obesity or unhealthy living conditions, has been considered in computational models via homogenization approach on the lobular scale (Ricken et al., 2018; Lambers et al., 2019, 2021; Ricken and Lambers, 2019), simulating fat accumulation and tissue repair (Holzhütter and Berndt, 2020), via ML (Deo and Panigrahi, 2019), focusing on zonated fat accumulation (Ashworth et al., 2016) or to investigate the influence of underlying metabolic processes (Naik et al., 2014; Wallstab et al., 2017; Maldonado et al., 2018). Detoxification of drugs and toxins is one of the main functions of the human liver. Computational models can help to predict detoxification of acetaminophen (Diaz Ochoa et al., 2012; Sluka et al., 2016; Lambers et al., 2018), ammonia and $\mathrm{CCl}_{4}$ (Schliess et al., 2014). As a consequence of many liver diseases, such as fibrosis or NAFLD, liver cancer can develop by forming tumors in liver tissue. In order to better investigate the development and spread of tumors in the liver, mathematical models can be used. These models primarily differentiate between primary liver tumors such as processes in hepatocellular carcinoma (HCC) (Berndt et al., 2019) and the development of liver tumors through metastases, for example from the colon (Wang et al., 2020). For the identification and prognosis of liver cancer, ML approaches (Keshavarz and Mojra, 2015; Chaudhary et al., 2018; Zhang et al., 2020) are also used, which can map the disease pattern using clinical and experimental data. Mathematical models can also be used to better understand the treatment options for liver tumors. For example, drug-based cancer nanotherapy (Frieboes et al., 2020) or radioembolization dosimetry (Taebi et al., 2020) can be examined for their effectiveness. Although there are already many developed models for the simulation of hepatic processes, some liver diseases are not yet represented by mathematical or numerical models. While some models focus on the mathematical description of hepatitis B and C, no model has yet been developed to describe the underlying processes of hepatitis A. Only models for the epidemiological spread of this disease have been developed to date (van Effelterre et al., 2017). Iberogast $\AA$, a widespread over-the-counter herbal product for gastrointestinal complaints, is suspected of having negative effects on liver function and of already being accountable for acute liver failure in one case (Gerhardt et al., 2019). The celandine contained therein is said to be the responsible cause.

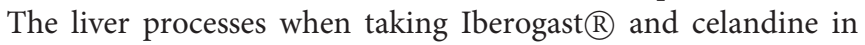
general as well as possible predictions and therapies of liver damage have not yet been described mathematically. 


\subsection{MRI-Based Simulations}

Several mathematical models based on MRI data have been developed in recent years. To enable a more detailed and patientspecific simulation of hepatic processes, models of the total organ can be coupled with simulations of MRI processes (Mescam et al., 2009). Furthermore, MRI data can be used to include patientspecific information into the models to better represent realistic conditions within the liver. For example, it is possible to simulate hepatic flow based on MRI images in healthy (Stoter et al., 2017; Rutkowski et al., 2019) or cirrhotic patients (Rutkowski et al., 2019). MRI techniques and measurements can be improved with the help of computational modeling, e.g., by using simulations of hepatic blood flow, and different hypotheses for finding new important disease parameters in MRI measurements can be tested in silico (George et al., 2015). Since liver transplantation remains the only curative treatment for several liver diseases like malignant liver tumors, a virtual surgery planning helps to improve the construction of hepatectomy in living liver donors during transplantation (Rutkowski et al., 2018). Here, MRI can determine the hemodynamics before and after surgery to validate the surgical planning tool. Also, microscopic hepatic surgeries can be simulated with mathematical models based on MRI data. To combine preoperative data and the resulting deformation of the organ during surgery, computational models can help to improve the liver surgery (Plantefève et al., 2016). Additionally, simulation is used to interpret and analyze MRI data. Therefore, hepatic parameters can be extracted from imaging data and can be used to predict liver function (Forsgren et al., 2014).

\subsection{Computational Models in Clinical Practice}

The main objective of the validated computer-controlled models is their application in clinical practice. To date, there are a few models that are already successfully used by physicians and medical companies to simulate and visualize hepatic processes. Several commercial tools are available to visualize the patient's anatomy using medical images (e.g., Myrian(c) from Intrasense, Ziostation $(\subset$ from Ziosoft, Synapse $\odot$ Vincent from Fujinon, Iqqa $\odot$ Liver from Edda Technology, Scout ${ }^{\mathrm{TM}}$ Liver from Pathfinder). These programs focus only on medical images neglecting function or perfusion calculation of the liver as well as the individual material behavior. To successfully design a planned operation in advance, programs can be applied to provide a three-dimensional reconstruction of the human anatomy from patient data using virtual reality (Reitinger et al., 2006; Soler et al., 2014) or image segmentation (Schenk et al., 1999; MeVis, 2020). In order to account for changes in the surgical requirements, additional effects such as deformations (Oshiro et al., 2015), material behavior (Marchesseau et al., 2010), and hemodynamics (Rutkowski et al., 2018) can also be taken into consideration.

\subsection{Strength and Weakness of the Review}

Based on our best knowledge, our review is the first systematic review for MRI-based liver biomechanics. Due to the limitations of the search platforms in the engineering fields, we had to base our main search strategy on Ovid Medline and Web of Science, the main search engines in the medical field. While it helped us perform a comprehensive search, this process could not be repeated completely in ScienceDirect, Wiley Online Library, and Taylor \& Francis, the fundamental search platforms for engineering fields. Another aspect of our review, which we believe should be seen as a strength and could be a lead for future similar reviews, is using a quality assessment tool based on standard protocols. However, due to the revisions that we had to make, future changes would also be possible. Moreover, future researches will also highlight the need for this kind of assessment, and further revisions will eventually result in standard assessment tools in this field. While one of our objectives was to comment on the best viscoelastic model for various MRE clinical applications, we could not make such recommendations due to the lack of sufficient evidence. Besides proposing the best viscoelastic model for clinical applications of MRE, one of our primary objectives was to assess the quality of the included papers. The results of the assessment, besides a lack of enough evidence for the proposed clinical application, highlight the need for better research designs in the future. A major part of quality assessment is considered to be the population or, in this case, the objects to be studied. Most of the studies were not successful in recruiting enough healthy volunteers or patient subjects for their research, which, therefore, does not represent the target population. To meet the demands of the clinical setting on liver biomechanics, more attention should be paid to participant selection, recruitment, and data collection.

\section{CONCLUSION}

In this review, recent advances in MRI-based biomechanics for the liver were discussed and summarized. The study focused on the liver MRE, viscoelastic constitutive models and material parameters, and modeling used for liver simulation, the possible clinical applications of these papers, and the studied diseases. Furthermore, the application of liver MRE and simulation for diagnosing liver disease and treatment processes not studied in the papers is discussed. Computational modeling and simulation provide descriptive and predictive tools to identify multiscale interactions and lead to a better understanding of healthy and diseased liver function, potentially in an individualized manner. Despite sophisticated modeling development, liver simulations consistently lag behind in terms of structural knowledge, that is, knowledge of the relationship between structure and function within the liver. The results of liver MRI can fill this gap. This increases the potential to explain the role of the liver and incorporate a technical understanding of the relationship between liver structural changes in diseases. Due to the lack of the best constitutive models for various MRE techniques, researchers may consider the impact of constitutive models on simulating liver disease. In the future, research groups should integrate various ML and MRI data extraction aspects to improve the biomechanical and clinical applications of MRI in liver studies. As a biomechanical application, the artificial neural network (ANN)-based constitutive model possesses the abilities of adjustment, memorization, anticipation, and better performances than the conventional constitutive equations. 
Therefore, ANNs can provide a novel approach to materials modeling, especially for complex and nonlinear relationships. Furthermore, DL algorithms, including convolutional neural network $(\mathrm{CNN})$, demonstrate strong diagnosis, and grading application of liver diseases such as fibrosis staging, diagnosis of fatty liver, and detection and classification of tumors. In addition, researchers must also seek to achieve reproducibility, validation, and verification of robust models.

\section{DATA AVAILABILITY STATEMENT}

The original contributions presented in the study are included in the article/Supplementary Material, further inquiries can be directed to the corresponding author/s.

\section{AUTHOR CONTRIBUTIONS}

SS devised the project, the main conceptual ideas, and proof outline. H-MT, IS, JR, and TR helped supervise the project. SS and $\mathrm{MN}$ worked out review of the papers with the help from SN

\section{REFERENCES}

Ahmadi-Badejani, R., Mosharaf-Dehkordi, M., and Ahmadikia, H. (2020). An image-based geometric model for numerical simulation of blood perfusion within the liver lobules. Comput. Methods Biomech. Biomed. Eng. 23, 987-1004. doi: 10.1080/10255842.2020.1782389

Amili, O., Golzarian, J., and Coletti, F. (2019). In vitro study of particle transport in successively bifurcating vessels. Ann. Biomed. Eng. 47, 2271-2283. doi: 10.1007/s10439-019-02293-2

Angulo, P., Hui, J. M., Marchesini, G., Bugianesi, E., George, J., Farrell, G. C., et al. (2007). The NAFLD fibrosis score: a noninvasive system that identifies liver fibrosis in patients with NAFLD. Hepatology 45, 846-854. doi: $10.1002 /$ hep. 21496

Asbach, P., Klatt, D., Hamhaber, U., Braun, J., Somasundaram, R., Hamm, B., et al. (2008). Assessment of liver viscoelasticity using multifrequency MR elastography. Magn. Reson. Med. 60, 373-379. doi: 10.1002/mrm.21636

Asbach, P., Klatt, D., Schlosser, B., Biermer, M., Muche, M., Rieger, A., et al. (2010). Viscoelasticity-based staging of hepatic fibrosis with multifrequency MR elastography. Radiology 257, 80-86. doi: 10.1148/radiol.10092489

Asbach, P., Ro, S.-R., Aldoj, N., Snellings, J., Reiter, R., Lenk, J., et al. (2020). In vivo quantification of water diffusion, stiffness, and tissue fluidity in benign prostatic hyperplasia and prostate cancer. Invest. Radiol. 55, 524-530. doi: 10.1097/RLI.0000000000000685

Ashworth, W. B., Davies, N. A., and Bogle, I. D. L. (2016). A computational model of hepatic energy metabolism: understanding zonated damage and steatosis in NAFLD. PLoS Comput. Biol. 12:e1005105. doi: 10.1371/journal.pcbi.1005105

Bane, O., Peti, S., Wagner, M., Hectors, S., Dyvorne, H., Markl, M., et al. (2019). Hemodynamic measurements with an abdominal $4 \mathrm{~d}$ flow MRI sequence with spiral sampling and compressed sensing in patients with chronic liver disease. J. Magn. Reson. Imag. 49, 994-1005. doi: 10.1002/jmri.26305

Barr, R. G. (2018). Liver elastography still in its infancy. Radiology 288, 107-108. doi: 10.1148/radiol.2018180777

Berndt, N., Eckstein, J., Heucke, N., Gajowski, R., Stockmann, M., Meierhofer, D., et al. (2019). Characterization of lipid and lipid droplet metabolism in human HCC. Cells 8, 1-18. doi: 10.3390/cells8050512

Bonekamp, S., Kamel, I., Solga, S., and Clark, J. (2009). Can imaging modalities diagnose and stage hepatic fibrosis and cirrhosis accurately? J. Hepatol. 50, 17-35. doi: 10.1016/j.jhep.2008.10.016

Brock, K., Sharpe, M., Dawson, L., Kim, S., and Jaffray, D. (2005). Accuracy of finite element model-based multi-organ deformable image registration. Med. Phys. 32(6 Pt 1), 1647-1659. doi: 10.1118/1.1915012 and LL. SS wrote the manuscript with input from all authors. All authors provided critical feedback and helped shape the research, analysis and manuscript.

\section{FUNDING}

This work was funded by Deutsche Forschungsgemeinschaft (DFG, German Research Foundation) under Germany's Excellence Strategy-EXC 2075-390740016, the Research Unit Programme FOR 5151 QuaLiPerF (Quantifying Liver Perfusion-Function Relationship in Complex Resection-A Systems Medicine Approach) by grant number 436883643 and the SPP 2311 SIMulation supported LIVer Assessment for donor organs (SimLivA) by grant number 465194077.

\section{SUPPLEMENTARY MATERIAL}

The Supplementary Material for this article can be found online at: https://www.frontiersin.org/articles/10.3389/fphys. 2021.733393/full\#supplementary-material

Campo, A., Dudzik, G., Apostolakis, J., Waz, A., Nauleau, P., Abramski, K., et al. (2017). Comparison between multi-channel LDV and PWI for measurement of pulse wave velocity in distensible tubes: towards a new diagnostic technique for detection of arteriosclerosis. Opt. Lasers Eng. 97, 41-51. doi: 10.1016/j.optlaseng.2017.05.013

Castera, L., Forns, X., and Alberti, A. (2008). Non-invasive evaluation of liver fibrosis using transient elastography. J. Hepatol. 48, 835-847. doi: 10.1016/j.jhep.2008.02.008

Catheline, S., Gennisson, J.-L., Delon, G., Fink, M., Sinkus, R., Abouelkaram, S., et al. (2004). Measurement of viscoelastic properties of homogeneous soft solid using transient elastography: an inverse problem approach. J. Acoust. Soc. Am. 116, 3734-3741. doi: 10.1121/1.1815075

Chaudhary, K., Poirion, O. B., Lu, L., and Garmire, L. X. (2018). Deep learningbased multi-omics integration robustly predicts survival in liver cancer. Clin. Cancer Res. 24, 1248-1259. doi: 10.1158/1078-0432.CCR-17-0853

Chen, J., Talwalkar, J. A., Yin, M., Glaser, K. J., Sanderson, S. O., and Ehman, R. L. (2011). Early detection of nonalcoholic steatohepatitis in patients with nonalcoholic fatty liver disease by using MR elastography. Radiology 259, 749-756. doi: 10.1148/radiol.11101942

Chen, Y., Ruan, D., Xiao, J., Wang, L., Sun, B., Saouaf, R., et al. (2020). Fully automated multi-organ segmentation in abdominal magnetic resonance imaging with deep neural networks. Med. Phys. 47:4971. doi: 10.1002/mp. 14429

Chlebus, G., Schenk, A., Moltz, J. H., van Ginneken, B., Hahn, H. K., and Meine, H. (2018). Automatic liver tumor segmentation in CT with fully convolutional neural networks and object-based postprocessing. Sci. Rep. 8, 1-7. doi: 10.1038/s41598-018-33860-7

Cieciura, T., Hryniewiecka, E., Perkowska-Ptasińska, A., Ciszek, M., and Pączek, L. (2020). Shear wave elastography performance in noninvasive assessment of liver cirrhosis in liver transplant recipients with the recurrence of hepatitis c infection. Transpl. Proc. 52, 2480-2483. doi: 10.1016/j.transproceed.2020.02.097

Clarke, E., Cheng, S., Green, M., Sinkus, R., and Bilston, L. (2011). Using static preload with magnetic resonance elastography to estimate large strain viscoelastic properties of bovine liver. J. Biomech. 44, 2461-2465. doi: 10.1016/j.jbiomech.2011.06.023

Courtecuisse, H., Peterlik, I., Trivisonne, R., Duriez, C., and Cotin, S. (2014). Constraint-based simulation for non-rigid real-time registration. Med. Meets Virt. Real. 196, 76-82. doi: 10.1007/978-3-319-07521-1_21

de Boer, R. (2012). Theory of Porous Media: Highlights in Historical Development and Current State. Berlin: Springer Science \& Business Media. 
Deo, R., and Panigrahi, S. (2019). "Prediction of hepatic steatosis (fatty liver) using machine learning," in Proceedings of the 2019 3rd International Conference on Computational Biology and Bioinformatics - ICCBB'19 (New York, NY: ACM Press), 8-12. doi: 10.1145/3365966.3365968

Diaz Ochoa, J. G., Bucher, J., Péry, A. R. R., Zaldivar Comenges, J. M., Niklas, J., and Mauch, K. (2012). A multi-scale modeling framework for individualized, spatiotemporal prediction of drug effects and toxicological risk. Front. Pharmacol. 3:204. doi: 10.3389/fphar.2012.00204

Drumheller, D. S. (1978). The theoretical treatment of a porous solid using a mixture theory. Int. J. Solids Struct. 14, 441-456. doi: 10.1016/0020-7683(78)90009-4

Dutta-Moscato, J., Solovyev, A., Mi, Q., Nishikawa, T., Soto-Gutierrez, A., Fox, I. J., et al. (2014). A multiscale agent-based in silico model of liver fibrosis progression. Front. Bioeng. Biotechnol. 2:18. doi: 10.3389/fbioe.2014.00018

Dzyubak, B., Li, J., Chen, J., Mara, K. C., Therneau, T. M., Venkatesh, S. K., et al. (2021). Automated analysis of multiparametric magnetic resonance imaging/magnetic resonance elastography exams for prediction of nonalcoholic steatohepatitis. J. Magn. Reson. Imag. 54, 122-131. doi: $10.1002 /$ jmri.27549

Eaton, J. E., Sen, A., Hoodeshenas, S., Schleck, C. D., Harmsen, W. S., Gores, G. J., et al. (2020). Changes in liver stiffness, measured by magnetic resonance elastography, associated with hepatic decompensation in patients with primary sclerosing cholangitis. Clin. Gastroenterol. Hepatol. 18, 1576-1583. doi: 10.1016/j.cgh.2019.10.041

Elias, D., Baton, O., Sideris, L., Boige, V., Malka, D., Liberale, G., et al. (2005). Hepatectomy plus intraoperative radiofrequency ablation and chemotherapy to treat technically unresectable multiple colorectal liver metastases. J. Surg. Oncol. 90, 36-42. doi: 10.1002/jso.20237

Evans, A., Sim, Y. T., Lawson, B., and Whelehan, P. (2020). Audit of eliminating biopsy for presumed fibroadenomas with benign ultrasound greyscale and shear-wave elastography findings in women aged 25-39 years. Clin. Radiol. 75, 880.e1-880.e3. doi: 10.1016/j.crad.2020.08.002

Evans, A., Whelehan, P., Thomson, K., McLean, D., Brauer, K., Purdie, C., et al. (2010). Quantitative shear wave ultrasound elastography: initial experience in solid breast masses. Breast Cancer Res. 12:R104. doi: 10.1186/bcr2787

Fang, C., and Sidhu, P. S. (2020). Ultrasound-based liver elastography: current results and future perspectives. Abdom. Radiol. 45, 3463-3472. doi: $10.1007 /$ s00261-020-02717-x

Faria, S. C., Ganesan, K., Mwangi, I., Shiehmorteza, M., Viamonte, B., Mazhar, S., et al. (2009). MR imaging of liver fibrosis: current state of the art. Radiographics 29, 1615-1635. doi: 10.1148/rg.296095512

Fischer, T., Sack, I., and Thomas, A. (2013). "Characterization of focal breast lesions by means of elastography," in RöFo-Fortschritte auf dem Gebiet der Röntgenstrahlen und der bildgebenden Verfahren, Vol. 185 (Stuttgart; New York, NY: Georg Thieme Verlag KG), 816-823. doi: 10.1055/s-00331335939

Ford, M. R., Dupps, W. J., Rollins, A. M., Roy, A. S., and Hu, Z. (2011). Method for optical coherence elastography of the cornea. J. Biomed. Opt. 16, 16005-16007. doi: $10.1117 / 1.3526701$

Forsgren, M. F., Dahlqvist Leinhard, O., Dahlström, N., Cedersund, G., and Lundberg, P. (2014). Physiologically realistic and validated mathematical liver model reveals corrected hepatobiliary transfer rates for Gd-EOB-DTPA using human DCE-MRI data. PLoS ONE 9:e95700. doi: 10.1371/journal.pone.0095700

Frieboes, H. B., Raghavan, S., and Godin, B. (2020). Modeling of nanotherapy response as a function of the tumor microenvironment: focus on liver metastasis. Front. Bioeng. Biotechnol. 8:1011. doi: 10.3389/fbioe.2020.01011

Friedman, A., and Hao, W. (2017). Mathematical modeling of liver fibrosis. Math. Biosci. Eng. 14, 143-164. doi: 10.3934/mbe.2017010

Gao, L., Parker, K., Lerner, R., and Levinson, S. (1996). Imaging of the elastic properties of tissue-a review. Ultrasound Med. Biol. 22, 959-977. doi: 10.1016/S0301-5629(96)00120-2

Garcia, S. R. M., Grossmann, M., Bruns, A., Dürr, M., Tzschätzsch, H., Hamm, B., et al. (2019). Tomoelastography paired with $\mathrm{t} 2^{\star}$ magnetic resonance imaging detects lupus nephritis with normal renal function. Invest. Radiol. 54, 89-97. doi: 10.1097/RLI.0000000000000511

Garteiser, P., Doblas, S., Daire, J.-L., Wagner, M., Leitao, H., Vilgrain, V., et al. (2012). MR elastography of liver tumours: value of viscoelastic properties for tumour characterisation. Eur. Radiol. 22, 2169-2177. doi: 10.1007/s00330-012-2474-6

George, S. M., Eckert, L. M., Martin, D. R., and Giddens, D. P. (2015). Hemodynamics in normal and diseased livers: application of imagebased computational models. Cardiovasc. Eng. Technol. 6, 80-91. doi: 10.1007/s13239-014-0195-5

Gerhardt, F., Benesic, A., Tillmann, H. L., Rademacher, S., Wittekind, C., Gerbes, A. L., et al. (2019). Iberogast-induced acute liver failure-reexposure and in vitro assay support causality. Off. J. Am. Coll. Gastroenterol. 114, 1358-1359. doi: 10.14309/ajg.0000000000000300

Gidener, T., Ahmed, O. T., Larson, J. J., Mara, K. C., Therneau, T. M., Venkatesh, S. K., et al. (2020). Liver stiffness by magnetic resonance elastography predicts future cirrhosis, decompensation, and death in NAFLD. Clin. Gastroenterol. Hepatol. 19, 1915-1924.e6. doi: 10.1016/j.cgh.2020.09.044

Godfrey, E. M., Patterson, A. J., Priest, A. N., Davies, S. E., Joubert, I., Krishnan, A. S., et al. (2012). A comparison of MR elastography and $31 \mathrm{p}$ MR spectroscopy with histological staging of liver fibrosis. Eur. Radiol. 22, 2790-2797. doi: 10.1007/s00330-012-2527-x

Goldaracena, N., and Barbas, A. S. (2019). Living donor liver transplantation. Curr. Opin. Organ Transpl. 24, 131-137. doi: 10.1097/MOT.0000000000000610

Gotra, A., Sivakumaran, L., Chartrand, G., Vu, K.-N., Vandenbroucke-Menu, F., Kauffmann, C., et al. (2017). Liver segmentation: indications, techniques and future directions. Insights Imaging 8, 377-392. doi: 10.1007/s13244-017-0558-1

Hariharan, P., Chang, I., Myers, M. R., and Banerjee, R. K. (2007). Radiofrequency ablation in a realistic reconstructed hepatic tissue. J. Biomech. Eng. 129, 354-364. doi: 10.1115/1.2720912

Hariharan, P., Freed, M., and Myers, M. R. (2013). Use of computational fluid dynamics in the design of dynamic contrast enhanced imaging phantoms. Phys. Med. Biol. 58:6369. doi: 10.1088/0031-9155/58/18/6369

Harrison, S. A., Oliver, D., Arnold, H. L., Gogia, S., and Neuschwander-Tetri, B. A. (2008). Development and validation of a simple NAFLD clinical scoring system for identifying patients without advanced disease. Gut 57, 1441-1447. doi: 10.1136/gut.2007.146019

Higgins, J. P., Thomas, J., Chandler, J., Cumpston, M., Li, T., Page, M. J., et al. (2019). Cochrane Handbook for Systematic Reviews of Interventions. John Wiley \& Sons. doi: 10.1002/9781119536604

Hindle, B. R., Lorimer, A., Winwood, P., and Keogh, J. W. (2019). The biomechanics and applications of strongman exercises: a systematic review. Sports Med. Open 5, 1-19. doi: 10.1186/s40798-019-0222-z

Hirsch, S., Braun, J., and Sack, I. (2017). Magnetic Resonance Elastography: Physical Background and Medical Applications. Weinheim: John Wiley \& Sons. doi: $10.1002 / 9783527696017$

Hoang, P., Wallace, A., Sugi, M., Fleck, A., Pershad, Y., Dahiya, N., et al. (2017). Elastography techniques in the evaluation of deep vein thrombosis. Cardiovasc. Diagn. Ther. 7(Suppl. 3), 238-245. doi: 10.21037/cdt.2017.10.04

Holm, S. (2019). Waves With Power-Law Attenuation. Cham: Springer. doi: 10.1007/978-3-030-14927-7

Holzhütter, H.-G., and Berndt, N. (2020). Mathematical modeling of free fatty acid-induced non-alcoholic fatty liver disease (NAFLD). bioRxiv 11, 1-22. doi: $10.1101 / 2020.05 .28 .120626$

Hu, J., Lü, S., Feng, S., and Long, M. (2017). Flow dynamics analyses of pathophysiological liver lobules using porous media theory. Acta Mech. Sin. 33, 823-832. doi: 10.1007/s10409-017-0674-7

Hudert, C. A., Tzschätzsch, H., Rudolph, B., Bläker, H., Loddenkemper, C., Müller, H.-P., et al. (2019). Tomoelastography for the evaluation of pediatric nonalcoholic fatty liver disease. Invest. Radiol. 54, 198-203. doi: 10.1097/RLI.0000000000000529

Idkaidek, A., and Jasiuk, I. (2015). Toward high-speed 3d nonlinear soft tissue deformation simulations using abaqus software. J. Robot. Surg. 9, 299-310. doi: 10.1007/s11701-015-0531-2

Imajo, K., Kessoku, T., Honda, Y., Tomeno, W., Ogawa, Y., Mawatari, H., et al. (2016). Magnetic resonance imaging more accurately classifies steatosis and fibrosis in patients with nonalcoholic fatty liver disease than transient elastography. Gastroenterology 150, 626-637. doi: 10.1053/j.gastro.2015.11.048

Iwai, M., Kojima, T., and Suriawinata, A. A. (2019). Anatomy and Function. Singapore: Springer Singapore. doi: 10.1007/978-981-13-6806-6_1

Kamphues, C., Klatt, D., Bova, R., Yahyazadeh, A., Bahra, M., Braun, J., et al. (2012). Viscoelasticity-based magnetic resonance elastography for the assessment of 
liver fibrosis in hepatitis C patients after liver transplantation. Rofo 184, 1013-1019. doi: 10.1055/s-0032-1313126

Keshavarz, M., and Mojra, A. (2015). Geometrical features assessment of liver's tumor with application of artificial neural network evolved by imperialist competitive algorithm. Int. J. Numer. Methods Biomed. Eng. 31:e02704. doi: $10.1002 / \mathrm{cnm} .2704$

Klatt, D., Hamhaber, U., Asbach, P., Braun, J., and Sack, I. (2007). Noninvasive assessment of the rheological behavior of human organs using multifrequency MR elastography: a study of brain and liver viscoelasticity. Phys. Med. Biol. 52, 7281-7294. doi: 10.1088/0031-9155/52/24/006

Klisch, S. M., Chen, S. S., Sah, R. L., and Hoger, A. (2003). A growth mixture theory for cartilage with application to growth-related experiments on cartilage explants. J. Biomech. Eng. 125, 169-179. doi: 10.1115/1.1560144

Koeppen, B. M., and Stanton, B. A. (2017). Berne and Levy Physiology e-book. Elsevier Health Sciences.

Koizumi, Y., Hirooka, M., Kisaka, Y., Konishi, I., Abe, M., Murakami, H., et al. (2011). Liver fibrosis in patients with chronic hepatitis c: noninvasive diagnosis by means of real-time tissue elastography-establishment of the method for measurement. Radiology 258, 610-617. doi: 10.1148/radiol.10100319

Kolipaka, A., Woodrum, D., Araoz, P. A., and Ehman, R. L. (2012). MR elastography of the in vivo abdominal aorta: a feasibility study for comparing aortic stiffness between hypertensives and normotensives. J. Magn. Reson. Imaging 35, 582-586. doi: 10.1002/jmri.22866

Konar, M., and Lang, J. (2011). Pros and cons of low-field magnetic resonance imaging in veterinary practice. Vet. Radiol. Ultrasound 52, S5-S14. doi: 10.1111/j.1740-8261.2010.01780.x

Kruse, S., Smith, J., Lawrence, A., Dresner, M., Manduca, A., Greenleaf, J. F., et al. (2000). Tissue characterization using magnetic resonance elastography: preliminary results. Phys. Med. Biol. 45, 1579-1590. doi: 10.1088/0031-9155/45/6/313

Lambers, L., Ricken, T., and Köng, M. (2019). “A multiscale and multiphase model for the description of function-perfusion processes in the human liver," in Advances in Engineering Materials, Structures and Systems: Innovations, Mechanics and Applications, ed A. Zingoni (London: CRC Press), 304-307. doi: 10.1201/9780429426506-52

Lambers, L., Suditsch, M., Wagner, A., and Ricken, T. (2021). A multiscale and multiphase model of function-perfusion growth processes in the human liver. PAMM 20:e202000290. doi: 10.1002/pamm.202000290

Lambers, L., Waschinsky, N., and Ricken, T. (2018). On a multi-scale and multiphase model of paracetamol-induced hepatotoxicity for human liver. PAMM 18:e201800454. doi: 10.1002/pamm.201800454

Lan, G., Gu, B., Larin, K. V., and Twa, M. D. (2020). Clinical corneal optical coherence elastography measurement precision: effect of heartbeat and respiration. Transl. Vis. Sci. Technol. 9:3. doi: 10.1167/tvst.9.5.3

Lang, S. T., Guo, J., Bruns, A., Dürr, M., Braun, J., Hamm, B., et al. (2019). Multiparametric quantitative MRI for the detection of IGA nephropathy using tomoelastography, DWI, and bold imaging. Invest. Radiol. 54, 669-674. doi: 10.1097/RLI.0000000000000585

Lara, J., López-Labrador, F., González-Candelas, F., Berenguer, M., and Khudyakov, Y. E. (2014). Computational models of liver fibrosis progression for hepatitis c virus chronic infection. BMC Bioinformatics 15(Suppl. 8), 1-9. doi: 10.1186/1471-2105-15-S8-S5

Lara, M., Chen, C.-Y., Mannor, P., Dur, O., Menon, P. G., Yoganathan, A. P., et al. (2011). Hemodynamics of the hepatic venous three-vessel confluences using particle image velocimetry. Ann. Biomed. Eng. 39, 2398-2416. doi: 10.1007/s10439-011-0326-1

Lautt, W. W. (2009). "Hepatic circulation: physiology and pathophysiology," in Colloquium Series on Integrated Systems Physiology: From Molecule to Function, Vol. 1 (Morgan \& Claypool Publishers), 1-174. doi: 10.4199/C00004ED1V01Y200910ISP001

Lebre, M.-A., Arrouk, K., Văn, A.-K. V., Leborgne, A., Grand-Brochier, M., Beaurepaire, P., et al. (2017). "Medical image processing and numerical simulation for digital hepatic parenchymal blood flow," in International Workshop on Simulation and Synthesis in Medical Imaging (Cham: Springer), 99-108. doi: 10.1007/978-3-319-68127-6_11

Leclerc, G. E., Charleux, F., Ho Ba Tho, M.-C., and Bensamoun, S. F. (2015). Identification process based on shear wave propagation within a phantom using finite element modelling and magnetic resonance elastography. Comput. Methods Biomech. Biomed. Eng. 18, 485-491. doi: 10.1080/10255842.2013.818664

Leclerc, G. E., Charleux, F., Robert, L., Ho Ba Tho, M., Rhein, C., Latrive, J., et al. (2013). Analysis of liver viscosity behavior as a function of multifrequency magnetic resonance elastography (MMRE) postprocessing. J. Magn. Reson. Imag. 38, 422-428. doi: 10.1002/jmri.23986

Lee, S.-L., Chung, A., Lerotic, M., Hawkins, M. A., Tait, D., and Yang, G.-Z. (2010). "Dynamic shape instantiation for intra-operative guidance," in International Conference on Medical Image Computing and Computer-Assisted Intervention (Berlin: Springer), 69-76. doi: 10.1007/978-3-642-15705-9_9

Lee, Y. J., Lee, J. M., Lee, J. E., Lee, K. B., Lee, E. S., Yoon, J.-H., et al. (2014). Mr elastography for noninvasive assessment of hepatic fibrosis: reproducibility of the examination and reproducibility and repeatability of the liver stiffness value measurement. J. Magn. Reson. Imag. 39, 326-331. doi: 10.1002/jmri.24147

Li, H., Flé, G., Bhatt, M., Qu, Z., Ghazavi, S., Yazdani, L., et al. (2021). Viscoelasticity imaging of biological tissues and single cells using shear wave propagation. Front. Phys. 9:350. doi: 10.3389/fphy.2021.666192

Liu, Z., and Bilston, L. (2000). On the viscoelastic character of liver tissue: experiments and modelling of the linear behaviour. Biorheology 37, 191-201.

López-Mir, F., Naranjo, V., Angulo, J., Alca niz, M., and Luna, L. (2014). Liver segmentation in MRI: a fully automatic method based on stochastic partitions. Comput. Methods Prog. Biomed. 114, 11-28. doi: 10.1016/j.cmpb.2013.12.022

Low, G., Kruse, S. A., and Lomas, D. J. (2016). General review of magnetic resonance elastography. World J. Radiol. 8, 59-72. doi: 10.4329/wjr.v8.i1.59

Lu, Y.-C., and Untaroiu, C. D. (2014). A statistical geometrical description of the human liver for probabilistic occupant models. J. Biomech. 47, 3681-3688. doi: 10.1016/j.jbiomech.2014.09.031

Lupescu, I. G., Grasu, M. C., and Dumitru, R. L. (2020). "Mr. elastography and functional MRI of the liver," in Liver Diseases (Springer), 523-531. doi: 10.1007/978-3-030-24432-3_46

Ma, R., Hunter, P., Cousins, W., Ho, H., Bartlett, A., and Safaei, S. (2019). Anatomically based simulation of hepatic perfusion in the human liver. Int. J. Numer. Methods Biomed. Eng. 35:e3229. doi: 10.1002/cnm.3229

Malandrino, A., and Moeendarbary, E. (2019). Poroelasticity of living tissues. Encyclop. Biomed. Eng. 2, 238-245. doi: 10.1016/B978-0-12-801238-3.99932-X

Maldonado, E. M., Fisher, C. P., Mazzatti, D. J., Barber, A. L., Tindall, M. J., Plant, N. J., et al. (2018). Multi-scale, whole-system models of liver metabolic adaptation to fat and sugar in non-alcoholic fatty liver disease. NPJ Syst. Biol. Appl. 4, 1-10. doi: 10.1038/s41540-018-0070-3

Manduca, A., Bayly, P. J., Ehman, R. L., Kolipaka, A., Royston, T. J., Sack, I., et al. (2020). MR elastography: principles, guidelines, and terminology. Magn. Reson. Med. 85, 2377-2390. doi: 10.1002/mrm.28627

Manduca, A., Oliphant, T. E., Dresner, M. A., Mahowald, J., Kruse, S. A., Amromin, E., et al. (2001). Magnetic resonance elastography: noninvasive mapping of tissue elasticity. Med. Image Anal. 5, 237-254. doi: 10.1016/S1361-8415(00)00039-6

Marchesseau, S., Heimann, T., Chatelin, S., Willinger, R., and Delingette, H. (2010). Fast porous visco-hyperelastic soft tissue model for surgery simulation: application to liver surgery. Prog. Biophys. Mol. Biol. 103, 185-196. doi: 10.1016/j.pbiomolbio.2010.09.005

Mariappan, Y. K., Glaser, K. J., and Ehman, R. L. (2010). Magnetic resonance elastography: a review. Clin. Anat. 23, 497-511. doi: 10.1002/ca.21006

Mattei, G., and Ahluwalia, A. (2016). Sample, testing and analysis variables affecting liver mechanical properties: a review. Acta Biomater. 45, 60-71. doi: 10.1016/j.actbio.2016.08.055

McRobbie, D. W., Moore, E. A., Graves, M. J., and Prince, M. R. (2017). MRI from Picture to Proton. London: Cambridge University Press. doi: $10.1017 / 9781107706958$

Mescam, M., Kretowski, M., and Bezy-Wendling, J. (2009). Multiscale model of liver dce-mri towards a better understanding of tumor complexity. IEEE Trans. Med. Imaging 29, 699-707. doi: 10.1109/TMI.2009.2031435

MeVis (2020). LiverAnalyzer.

Meyers, M. A., and Chawla, K. K. (2008). Mechanical Behavior of Materials. Cambridge: Cambridge University Press. doi: 10.1017/CBO9780511810947

Mitchell, S., and Mendes, P. (2013). A computational model of liver iron metabolism. PLoS Comput. Biol. 9:e1003299. doi: 10.1371/journal.pcbi.1003299

Moawad, A. W., Szklaruk, J., Lall, C., Blair, K. J., Kaseb, A. O., Kamath, A., et al. (2020). Angiogenesis in hepatocellular carcinoma; pathophysiology, 
targeted therapy, and role of imaging. J. Hepatocell. Carcin. 7:77. doi: 10.2147/JHC.S224471

Monti, S., Palma, G., Ragucci, M., Mannelli, L., Mancini, M., and Prinster, A. (2014). Optimization of tagged MRI for quantification of liver stiffness using computer simulated data. PLOS ONE 9:e111852. doi: 10.1371/journal.pone.0111852

Motosugi, U., Roldán-Alzate, A., Bannas, P., Said, A., Kelly, S., Zea, R., et al. (2019). Four-dimensional flow MRI as a marker for risk stratification of gastroesophageal varices in patients with liver cirrhosis. Radiology 290, 101-107. doi: 10.1148/radiol.2018180230

Mueller, S. (2020). Liver Elastography - Clinical Use and Interpretation. Cham: Springer. doi: 10.1007/978-3-030-40542-7

Mumoli, N., Mastroiacovo, D., Giorgi-Pierfranceschi, M., Pesavento, R., Mochi, M., Cei, M., et al. (2018). Ultrasound elastography is useful to distinguish acute and chronic deep vein thrombosis. J. Thromb. Haemost. 16, 2482-2491. doi: $10.1111 /$ jth. 14297

Muthupillai, R., and Ehman, R. L. (1996). Magnetic resonance elastography. Nat. Med. 2, 601-603. doi: 10.1038/nm0596-601

Naik, A., Rozman, D., and Belič, A. (2014). Steatonet: the first integrated human metabolic model with multi-layered regulation to investigate liver-associated pathologies. PLoS Comput. Biol. 10:e1003993. doi: 10.1371/journal.pcbi.1003993

Netter, F. H. (2014). Atlas of Human Anatomy, Professional Edition E-Book: Including NetterReference. com Access With Full Downloadable Image Bank. Elsevier Health Sciences.

Ning, J., Schubert, T., Johnson, K. M., Roldán-Alzate, A., Chen, H., Yuan, C., et al. (2018). Vascular input function correction of inflow enhancement for improved pharmacokinetic modeling of liver DCE-MRI. Magn. Reson. Med. 79, 3093-3102. doi: 10.1002/mrm.26988

Ormachea, J., and Parker, K. J. (2020). Elastography imaging: the 30 year perspective. Phys. Med. Biol. 65:24TR06. doi: 10.1088/1361-6560/abca00

Oshiro, Y., Yano, H., Mitani, J., Kim, S., Kim, J., Fukunaga, K., et al. (2015). Novel 3-dimensional virtual hepatectomy simulation combined with real-time deformation. World J. Gastroenterol. 21, 9982-9992. doi: 10.3748/wjg.v21.i34.9982

Parker, K. (2014). A microchannel flow model for soft tissue elasticity. Phys. Med. Biol. 59:4443. doi: 10.1088/0031-9155/59/15/4443

Parker, K., Ormachea, J., Drage, M., Kim, H., and Hah, Z. (2018). The biomechanics of simple steatosis and steatohepatitis. Phys. Med. Biol. 63:105013. doi: 10.1088/1361-6560/aac09a

Parker, K., Ormachea, J., McAleavey, S., Wood, R., Carroll-Nellenback, J., and Miller, R. (2016). Shear wave dispersion behaviors of soft, vascularized tissues from the microchannel flow model. Phys. Med. Biol. 61:4890. doi: 10.1088/0031-9155/61/13/4890

Patki, P., and Costanzo, F. (2019). A mixture theory-based finite element formulation for the study of biodegradation of poroelastic scaffolds. Comput. Mech. 66, 351-371. doi: 10.1007/s00466-020-01854-w

Peeters, G., Debbaut, C., Cornillie, P., de Schryver, T., Monbaliu, D., Laleman, W., et al. (2015). A multilevel modeling framework to study hepatic perfusion characteristics in case of liver cirrhosis. J. Biomech. Eng. 137:051007. doi: 10.1115/1.4029280

Perri nez, P. R., Kennedy, F. E., Van Houten, E. E., Weaver, J. B., and Paulsen, K. D. (2008). Modeling of soft poroelastic tissue in time-harmonic MR elastography. IEEE Trans. Biomed. Eng. 56, 598-608. doi: 10.1109/TBME.2008.2009928

Perri nez, P. R., Pattison, A. J., Kennedy, F. E., Weaver, J. B., and Paulsen, K. D. (2010). Contrast detection in fluid-saturated media with magnetic resonance poroelastography. Med. Phys. 37(7 Pt 1), 3518-3526. doi: 10.1118/1.3443563

Planteféve, R., Peterlik, I., Haouchine, N., and Cotin, S. (2016). Patientspecific biomechanical modeling for guidance during minimally-invasive hepatic surgery. Ann. Biomed. Eng. 44, 139-153. doi: 10.1007/s10439-0151419-Z

Reiter, R., Freise, C., Jöhrens, K., Kamphues, C., Seehofer, D., Stockmann, M., et al. (2014). Wideband MRE and static mechanical indentation of human liver specimen: sensitivity of viscoelastic constants to the alteration of tissue structure in hepatic fibrosis. J. Biomech. 47, 1665-1674. doi: 10.1016/j.jbiomech.2014.02.034

Reiter, R., Tzschätzsch, H., Schwahofer, F., Haas, M., Bayerl, C., Muche, M., et al. (2020). Diagnostic performance of tomoelastography of the liver and spleen for staging hepatic fibrosis. Eur. Radiol. 30, 1719-1729. doi: 10.1007/s00330-019-06471-7

Reiter, R., Wetzel, M., Hamesch, K., Strnad, P., Asbach, P., Haas, M., et al. (2018). Comparison of non-invasive assessment of liver fibrosis in patients with alpha1antitrypsin deficiency using magnetic resonance elastography (MRE), acoustic radiation force impulse (ARFI) quantification, and 2d-shear wave elastography (2D-SWE). PLoS ONE 13:e0196486. doi: 10.1371/journal.pone.0196486

Reitinger, B., Bornik, A., Beichel, R., and Schmalstieg, D. (2006). Liver surgery planning using virtual reality. IEEE Comput. Graph. Appl. 26, 36-47. doi: 10.1109/MCG.2006.131

Ricken, T., and Lambers, L. (2019). On computational approaches of liver lobule function and perfusion simulation. GAMM-Mitteil. 42:e0198060. doi: 10.1002/gamm.201900016

Ricken, T., Waschinsky, N., and Werner, D. (2018). "Simulation of steatosis zonation in liver lobule-a continuummechanical bi-scale, tri-phasic, multicomponent approach," in Biomedical Technology, Vol. 84 of Lecture Notes in Applied and Computational Mechanics, eds P. Wriggers and T. Lenarz (Cham: Springer International Publishing), 15-33. doi: 10.1007/978-3-319-59548-1_2

Ricken, T., Werner, D., Holzhütter, H., König, M., Dahmen, U., and Dirsch, O. (2015). Modeling function-perfusion behavior in liver lobules including tissue, blood, glucose, lactate and glycogen by use of a coupled twoscale PDE?ODE approach. Biomech. Model. Mechanobiol. 14, 515-536. doi: 10.1007/s10237-014-0619-z

Riek, K., Klatt, D., Nuzha, H., Mueller, S., Neumann, U., Sack, I., et al. (2011). Wide-range dynamic magnetic resonance elastography. J. Biomech. 44, 1380-1386. doi: 10.1016/j.jbiomech.2010.12.031

Riva, F., Bisi, M., and Stagni, R. (2013). Orbital stability analysis in biomechanics: a systematic review of a nonlinear technique to detect instability of motor tasks. Gait Posture 37, 1-11. doi: 10.1016/j.gaitpost.2012.06.015

Roldán-Alzate, A., Frydrychowicz, A., Niespodzany, E., Landgraf, B. R., Johnson, K. M., Wieben, O., et al. (2013). In vivo validation of $4 \mathrm{D}$ flow MRI for assessing the hemodynamics of portal hypertension. J. Magn. Reson. Imag. 37, 1100-1108. doi: 10.1002/jmri.23906

Roldán-Alzate, A., Frydrychowicz, A., Said, A., Johnson, K. M., Francois, C. J., Wieben, O., et al. (2015). Impaired regulation of portal venous flow in response to a meal challenge as quantified by $4 \mathrm{D}$ flow MRI. J. Magn. Reson. Imag. 42, 1009-1017. doi: 10.1002/jmri.24886

Ronot, M., Lambert, S. A., Wagner, M., Garteiser, P., Doblas, S., Albuquerque, M., et al. (2014). Viscoelastic parameters for quantifying liver fibrosis: threedimensional multifrequency MR elastography study on thin liver rat slices. PLoS ONE 9:e94679. doi: 10.1371/journal.pone.0094679

Rutkowski, D. R., Medero, R., Garcia, F. J., and Roldán-Alzate, A. (2019). MRIbased modeling of spleno-mesenteric confluence flow. J. Biomech. 88, 95-103. doi: 10.1016/j.jbiomech.2019.03.025

Rutkowski, D. R., Reeder, S. B., Fernandez, L. A., and Roldán-Alzate, A. (2018). Surgical planning for living donor liver transplant using 4D flow MRI, computational fluid dynamics and in vitro experiments. Comput. Methods Biomech. Biomed. Eng. 6, 545-555. doi: 10.1080/21681163.2017.1278619

Sack, I., Beierbach, B., Hamhaber, U., Klatt, D., and Braun, J. (2008). Non-invasive measurement of brain viscoelasticity using magnetic resonance elastography. NMR Biomed. 21, 265-271. doi: 10.1002/nbm.1189

Sack, I., and Schaeffter, T. (2018). Quantification of Biophysical Parameters in Medical Imaging. Cham: Springer. doi: 10.1007/978-3-319-65924-4

Salameh, N., Larrat, B., Abarca-Quinones, J., Pallu, S., Dorvillius, M., Leclercq, I., et al. (2009). Early detection of steatohepatitis in fatty rat liver by using MR elastography. Radiology 253, 90-97. doi: 10.1148/radiol.2523 081817

Salameh, N., Peeters, F., Sinkus, R., Abarca-Quinones, J., Annet, L., Ter Beek, L. C. et al. (2007). Hepatic viscoelastic parameters measured with MR elastography: correlations with quantitative analysis of liver fibrosis in the rat. J. Magn. Reson. Imag. 26, 956-962. doi: 10.1002/jmri.21099

Sarvazyan, A., Hall, T., Urban, M., Fatemi, M., Aglyamov, S., and Garra, B. (2011). An overview of elastography-an emerging branch of medical imaging. Curr. Med. Imag. 7, 255-282. doi: 10.2174/157340511798038684

Schaafs, L.-A., Tzschätzsch, H., Reshetnik, A., van der Giet, M., Braun, J., Hamm, B., et al. (2019). Ultrasound time-harmonic elastography of the aorta: effect of age and hypertension on aortic stiffness. Invest. Radiol. 54, 675-680. doi: 10.1097/RLI.0000000000000590 
Scheinfeld, M. H., Bilali, A., and Koenigsberg, M. (2009). Understanding the spectral doppler waveform of the hepatic veins in health and disease. Radiographics 29, 2081-2098. doi: 10.1148/rg.297095715

Schenk, A., Breitenborn, J., Selle, D., Schindewolf, T., Böhm, D., Spindler, W., et al. (1999). "Ilabmed-workstation - eine entwicklungsumgebung für radiologische anwendungen," in Bildverarbeitung für die Medizin 1999, Informatik Aktuell, eds W. Brauer, H. Evers, G. Glombitza, H. P. Meinzer, and T. Lehmann (Berlin; Heidelberg: Springer Berlin Heidelberg), 238-242. doi: 10.1007/978-3-642-60125-5_44

Schliess, F., Hoehme, S., Henkel, S. G., Ghallab, A., Driesch, D., Böttger, J., et al. (2014). Integrated metabolic spatial-temporal model for the prediction of ammonia detoxification during liver damage and regeneration. Hepatology 60, 2040-2051. doi: 10.1002/hep.27136

Schwartz, J.-M., Denninger, M., Rancourt, D., Moisan, C., and Laurendeau, D. (2005). Modelling liver tissue properties using a non-linear viscoelastic model for surgery simulation. Med. Image Anal. 9, 103-112. doi: 10.1016/j.media.2004.11.002

Serai, S. D., Yin, M., Wang, H., Ehman, R. L., and Podberesky, D. J. (2015). Crossvendor validation of liver magnetic resonance elastography. Abdom. Imag. 40, 789-794. doi: 10.1007/s00261-014-0282-y

Shahryari, M., Tzschätzsch, H., Guo, J., Garcia, S. R. M., Böning, G., Fehrenbach, U., et al. (2019). Tomoelastography distinguishes noninvasively between benign and malignant liver lesions. Cancer Res. 79, 5704-5710. doi: 10.1158/0008-5472.CAN-19-2150

Shamseer, L., Moher, D., Clarke, M., Ghersi, D., Liberati, A., Petticrew, M., et al. (2015). Preferred reporting items for systematic review and meta-analysis protocols (PRISMA-P) 2015: elaboration and explanation. BMJ 349:g7647. doi: $10.1136 /$ bmj.g7647

Shiina, T., Nightingale, K. R., Palmeri, M. L., Hall, T. J., Bamber, J. C., Barr, R. G., et al. (2015). WFUMB guidelines and recommendations for clinical use of ultrasound elastography: Part 1: basic principles and terminology. Ultrasound Med. Biol. 41, 1126-1147. doi: 10.1016/j.ultrasmedbio.2015.03.009

Sluka, J. P., Fu, X., Swat, M., Belmonte, J. M., Cosmanescu, A., Clendenon, S. G., et al. (2016). A liver-centric multiscale modeling framework for xenobiotics. PLOS ONE 11:e0162428. doi: 10.1371/journal.pone.0162428

Soler, L., Nicolau, S., Pessaux, P., Mutter, D., and Marescaux, J. (2014). Real-time 3D image reconstruction guidance in liver resection surgery. Hepatobil. Surg. Nutr. 3, 73-81. doi: 10.3978/j.issn.2304-3881.2014.02.03

Sowinski, D. R., McGarry, M. D., Van Houten, E., Gordon-Wylie, S., Weaver, J., and Paulsen, K. D. (2020). Poroelasticity as a model of soft tissue structure: hydraulic permeability inference for magnetic resonance elastography in silico. arXiv preprint arXiv:2012.03993. doi: 10.3389/fphy.2020.617582

Srinivasa Babu, A., Wells, M. L., Teytelboym, O. M., Mackey, J. E., Miller, F. H., Yeh, B. M., et al. (2016). Elastography in chronic liver disease: modalities, techniques, limitations, and future directions. Radiographics 36, 1987-2006. doi: 10.1148/rg.2016160042

Stankovic, Z., Csatari, Z., Deibert, P., Euringer, W., Jung, B., Kreisel, W., et al. (2013). A feasibility study to evaluate splanchnic arterial and venous hemodynamics by flow-sensitive $4 \mathrm{~d}$ MRI compared with doppler ultrasound in patients with cirrhosis and controls. Eur. J. Gastroenterol. Hepatol. 25, 669-675. doi: 10.1097/MEG.0b013e32835e1297

Stankovic, Z., Jung, B., Collins, J., Russe, M. F., Carr, J., Euringer, W., et al. (2014). Reproducibility study of four-dimensional flow MRI of arterial and portal venous liver hemodynamics: influence of spatio-temporal resolution. Magn. Reson. Med. 72, 477-484. doi: 10.1002/mrm.24939

Stoter, S. K., Müller, P., Cicalese, L., Tuveri, M., Schillinger, D., and Hughes, T. J. (2017). A diffuse interface method for the navier-stokes/darcy equations: perfusion profile for a patient-specific human liver based on MRI scans. Comput. Methods Appl. Mech. Eng. 321, 70-102. doi: 10.1016/j.cma.2017. 04.002

Sumida, Y., Kamada, Y., Iwai, M., Kwo, P. Y., and Yoneda, M. (2019). "Laboratory tests in liver diseases," in Diagnosis of Liver Disease (Springer), 19-34. doi: 10.1007/978-981-13-6806-6_2

Sumida, Y., Yoneda, M., Hyogo, H., Yamaguchi, K., Ono, M., Fujii, H., et al. (2011). A simple clinical scoring system using ferritin, fasting insulin, and type IV collagen $7 \mathrm{~s}$ for predicting steatohepatitis in nonalcoholic fatty liver disease. J. Gastroenterol. 46, 257-268. doi: 10.1007/s00535-0100305-6
Taebi, A., Vu, C. T., and Roncali, E. (2020). Multi-scale computational fluid dynamics modeling for personalized liver cancer radioembolization dosimetry. J. Biomech. Eng. 143:011002. doi: 10.1115/1.4047656

Tan, L., McGarry, M. D., Van Houten, E. E., Ji, M., Solamen, L., Zeng, W., et al. (2017). A numerical framework for interstitial fluid pressure imaging in poroelastic MRE. PLoS ONE 12:e0178521. doi: 10.1371/journal.pone. 0178521

Tang, A., Cloutier, G., Szeverenyi, N. M., and Sirlin, C. B. (2015). Ultrasound elastography and MR elastography for assessing liver fibrosis: part 2, diagnostic performance, confounders, and future directions. Am. J. Roentgenol. 205, 33-40. doi: 10.2214/AJR.15.14553

Tang, W., and Wan, T. R. (2014). Constraint-based soft tissue simulation for virtual surgical training. IEEE Trans. Biomed. Eng. 61, 2698-2706. doi: 10.1109/TBME.2014.2326009

Thng, C. H., San Koh, T., Collins, D. J., and Koh, D. M. (2010). Perfusion magnetic resonance imaging of the liver. World J. Gastroenterol. 16:1598. doi: 10.3748/wjg.v16.i13.1598

Thomas, A., Fischer, T., Frey, H., Ohlinger, R., Grunwald, S., Blohmer, J.-U., et al. (2006). Real-time elastography-an advanced method of ultrasound: first results in 108 patients with breast lesions. Ultrasound Obstetr. Gynecol. 28, 335-340. doi: $10.1002 /$ uog. 2823

Tomita, S., Suzuki, H., Kajiwara, I., Nakamura, G., Jiang, Y., Suga, M., et al. (2018). Numerical simulations of magnetic resonance elastography using finite element analysis with a linear heterogeneous viscoelastic model. J. Visual. 21, 133-145. doi: 10.1007/s12650-017-0436-4

Trefts, E., Gannon, M., and Wasserman, D. H. (2017). The liver. Curr. Biol. 27, R1147-R1151. doi: 10.1016/j.cub.2017.09.019

Tzschätzsch, H., Guo, J., Dittmann, F., Hirsch, S., Barnhill, E., Jöhrens, K., et al. (2016). Tomoelastography by multifrequency wave number recovery from time-harmonic propagating shear waves. Med. Image Anal. 30, 1-10. doi: 10.1016/j.media.2016.01.001

Tzschätzsch, H., Ipek-Ugay, S., Guo, J., Streitberger, K.-J., Gentz, E., Fischer, T., et al. (2014). In vivo time-harmonic multifrequency elastography of the human liver. Phys. Med. Biol. 59, 1641-1654. doi: 10.1088/0031-9155/59/7/1641

Uman, L. S. (2011). Systematic reviews and meta-analyses. J. Can. Acad. Child Adolesc. Psychiatry 20, 57-59.

van Effelterre, T., Guignard, A., Marano, C., Rojas, R., and Jacobsen, K. H. (2017). Modeling the hepatitis a epidemiological transition in Brazil and Mexico. Hum. Vacc. Immunother. 13, 1942-1951. doi: 10.1080/21645515.2017.1323158

Vappou, J. (2012). Magnetic resonance- and ultrasound imaging- based elasticity imaging methods: a review. Crit. Rev. Biomed. Eng. 40, 121-134. doi: 10.1615/CritRevBiomedEng.v40.i2.30

Venkatesh, S. K., Yin, M., and Ehman, R. L. (2013). Magnetic resonance elastography of liver: technique, analysis, and clinical applications. J. Magn. Reson. Imaging 37, 544-555. doi: 10.1002/jmri.23731

Venkatesh, S. K., Yin, M., Takahashi, N., Glockner, J. F., Talwalkar, J. A., and Ehman, R. L. (2015). Non-invasive detection of liver fibrosis: MR imaging features vs. MR elastography. Abdom. Imag. 40, 766-775. doi: 10.1007/s00261-015-0347-6

Wallstab, C., Eleftheriadou, D., Schulz, T., Damm, G., Seehofer, D., Borlak, J., et al. (2017). A unifying mathematical model of lipid droplet metabolism reveals key molecular players in the development of hepatic steatosis. FEBS J. 284, 3245-3261. doi: 10.1111/febs.14189

Wang, S., and Larin, K. V. (2015). Optical coherence elastography for tissue characterization: a review. J. Biophoton. 8, 279-302. doi: $10.1002 /$ jbio. 201400108

Wang, Y., Brodin, E., Nishii, K., Frieboes, H. B., Mumenthaler, S., Sparks, J. L., et al. (2020). Impact of tumor-parenchyma biomechanics on liver metastatic progression: a multi-model approach. Sci. Rep. 11, 1-20. doi: 10.1038/s41598-020-78780-7

Wang, Y., Ganger, D. R., Levitsky, J., Sternick, L. A., McCarthy, R. J., Chen, Z. E., et al. (2011). Assessment of chronic hepatitis and fibrosis: comparison of MR elastography and diffusion-weighted imaging. Am. J. Roentgenol. 196, 553-561. doi: 10.2214/AJR.10.4580

Wei, W., Wu, X., Zhou, J., Sun, Y., Kong, Y., and Yang, X. (2019). Noninvasive evaluation of liver fibrosis reverse using artificial neural network model for chronic hepatitis B patients. Comput. Math. Methods Med. 2019:7239780. doi: $10.1155 / 2019 / 7239780$ 
Wells, P. N., and Liang, H.-D. (2011). Medical ultrasound: imaging of soft tissue strain and elasticity. J. R. Soc. Interface 8, 1521-1549. doi: 10.1098/rsif.2011.0054

Wiesemann, S., Schmitter, S., Demir, A., Prothmann, M., Schwenke, C., Chawla, A., et al. (2021). Impact of sequence type and field strength $(1.5,3$, and $7 \mathrm{t})$ on $4 \mathrm{~d}$ flow MRI hemodynamic aortic parameters in healthy volunteers. Magn. Reson. Med. 85, 721-733. doi: $10.1002 / \mathrm{mrm} .28450$

Yoneda, M., Fujita, K., Inamori, M., Nakajima, A., Tamano, M., and Hiraishi, H. (2007). Transient elastography in patients with non-alcoholic fatty liver disease (NAFLD). Gut 56, 1330-1331. doi: 10.1136/gut.2007.126417

Yoneda, M., Suzuki, K., Kato, S., Fujita, K., Nozaki, Y., Hosono, K., et al. (2010). Nonalcoholic fatty liver disease: us-based acoustic radiation force impulse elastography. Radiology 256, 640-647. doi: 10.1148/radiol.10091662

You, S. C., Kim, K. J., Kim, S. U., Kim, B. K., Park, J. Y., Kim, D. Y., et al. (2015). Hepatic fibrosis assessed using transient elastography independently associated with coronary artery calcification. J. Gastroenterol. Hepatol. 30, 1536-1542. doi: 10.1111/jgh.12992

Zhang, L., Yin, J., Duan, Y., Yang, Y., Yuan, L., and Cao, T. (2011). Assessment of intrahepatic blood flow by doppler ultrasonography: relationship between the hepatic vein, portal vein, hepatic artery and portal pressure measured intraoperatively in patients with portal hypertension. BMC Gastroenterol. 11:84. doi: 10.1186/1471-230X-11-84

Zhang, Y., Knopf, A., Tanner, C., Boye, D., and Lomax, A. J. (2013). Deformable motion reconstruction for scanned proton beam therapy using on-line $\mathrm{x}$-ray imaging. Phys. Med. Biol. 58, 8621-8645. doi: 10.1088/0031-9155/58/24/8621

Zhang, Y., Knopf, A., Tanner, C., and Lomax, A. J. (2014). Online image guided tumour tracking with scanned proton beams: a comprehensive simulation study. Phys. Med. Biol. 59, 7793-7817. doi: 10.1088/0031-9155/59/ $24 / 7793$
Zhang, Z.-M., Tan, J.-X., Wang, F., Dao, F.-Y., Zhang, Z.-Y., and Lin, H. (2020). Early diagnosis of hepatocellular carcinoma using machine learning method. Front. Bioeng. Biotechnol. 8:254. doi: 10.3389/fbioe.2020. 00254

Zhou, I. Y., Catalano, O. A., and Caravan, P. (2020). Advances in functional and molecular MRI technologies in chronic liver diseases. J. Hepatol. 73, P1241-1254. doi: 10.1016/j.jhep.2020.06.020

Zhu, L., Guo, J., Jin, Z., Xue, H., Dai, M., Zhang, W., et al. (2020). Distinguishing pancreatic cancer and autoimmune pancreatitis with in vivo tomoelastography. Eur. Radiol. 31, 3366-3374. doi: 10.1007/s00330-020-07420-5

Conflict of Interest: The authors declare that the research was conducted in the absence of any commercial or financial relationships that could be construed as a potential conflict of interest.

Publisher's Note: All claims expressed in this article are solely those of the authors and do not necessarily represent those of their affiliated organizations, or those of the publisher, the editors and the reviewers. Any product that may be evaluated in this article, or claim that may be made by its manufacturer, is not guaranteed or endorsed by the publisher.

Copyright (c) 2021 Seyedpour, Nabati, Lambers, Nafisi, Tautenhahn, Sack, Reichenbach and Ricken. This is an open-access article distributed under the terms of the Creative Commons Attribution License (CC BY). The use, distribution or reproduction in other forums is permitted, provided the original author(s) and the copyright owner(s) are credited and that the original publication in this journal is cited, in accordance with accepted academic practice. No use, distribution or reproduction is permitted which does not comply with these terms. 